\title{
Computational structure of a biological motion-detection system as revealed by local detector analysis in the fly's nervous system
}

\author{
Martin Egelhaaf, Alexander Borst, and Werner Reichardt \\ Max-Planck-Institut für Biologische Kybernetik, Spemannstrasse 38, D-7400 Tübingen, Federal Republic of \\ Germany
}

Received September 28, 1988; accepted February 6, 1989

\begin{abstract}
The computations performed by individual movement detectors are analyzed by intracellularly recording from an identified direction-selective motion-sensitive interneuron in the fly's brain and by comparing these results with model predictions based on movement detectors of the correlation type. Three main conclusions were drawn with respect to the movement-detection system of the fly: (1) The essential nonlinear interaction between the two movement-detector input channels can be characterized formally by a mathematically almost perfect multiplication process. (2) Even at high contrasts no significant nonlinearities seem to distort the time course of the movement-detector input signals. (3) The movement detectors of the fly are not perfectly antisymmetrical; i.e., they respond with different time courses and amplitudes to motion in their preferred and null directions. As a consequence of this property, the motion detectors can respond to some degree to stationary patterns whose brightness is modulated in time. Moreover, the direction selectivity, i.e., the relative difference of the responses to motion in the preferred and null directions, depends on the contrast and on the spatial-frequency content of the stimulus pattern.
\end{abstract}

\section{INTRODUCTION}

The direction and the velocity of a moving pattern are not represented explicitly at the level of the retinal input. Each photoreceptor, instead, provides information only on timedependent variations of local light intensity. From this input the visual system must compute, most likely in a series of processing steps, specific representations of motion information. In this study we shall be concerned with the process of motion detection, that is, the mechanisms that compute the initial explicit representation of motion information. A clear understanding of what information on the visual surround is represented by these initial motion measurements might be important, before the evaluation of the global aspects of the retinal motion patterns in tasks such as figure-ground separation or recovery of the three-dimensional shape of objects can be understood.

From a computational point of view, a motion-detection mechanism must satisfy at least three requirements ${ }^{1,2}$ : (1) It must have at least two input channels. Two inputs are necessary since motion is a vector in the spatiotemporal domain and a vector needs two points for its proper representation. (2) The underlying overall interaction between the two input channels must be nonlinear. This is because the time-averaged output of a linear interaction is identical to the result of the interaction of the time-averaged input signals. The time-averaged input signals do not preserve the information about the temporal order of the incoming stimuli. Therefore the information about the direction of motion is lost. (3) A movement detector must be asymmetrical in an appropriate way. If it were symmetrical, its two input channels could be interchanged. This would be equivalent to a reversal in the direction of motion. Without asymmetrical preprocessing the system would thus respond in the same way to motion in opposite directions.
These theoretical considerations provide us with only the essential requirements that must be met by any motiondetection system. However, they do not specify the nature of the essential nonlinear interaction of the movement-detector input signals or the representation of the visual environment on which this interaction operates. Evidence accumulating is now from the different disciplines in which biological motion information processing is studied, such as human psychophysics, electrophysiology of the vertebrate visual system, and invertebrate vision, that only a few partly equivalent mechanisms appear to be realized in the animal kingdom. Roughly speaking, they can be divided into two main categories, the so-called gradient and correlation models. The gradient model obtains a local-motion estimate by relating the simultaneously measured spatial and temporal changes in the local light intensity of the moving image. ${ }^{3-5}$ The gradient model was developed in the field of computer vision and, only later, was used to explain certain phenomena in motion perception of humans. 6,7 The so-called correlation model obtains a local-motion estimate by evaluating a kind of spatiotemporal cross correlation of the light intensity fluctuations at two neighboring points in the retinal image. 8,9 Its essential nonlinearity thus consists of a multiplicative interaction of two movement-detector input channels. The original correlation-type movement detector, ${ }^{8,9}$ as well as mathematically equivalent or slightly different versions of it, ${ }^{10-19}$ therefore represents two-input systems with a quadratic nonlinearity. This motion-detection scheme was deduced originally from behavioral experiments on insects. ${ }^{8,10,11,20-25}$ Subsequently, however, it was applied to various other biological motion vision systems in vertebrates including humans. ${ }^{13-19}$

Whereas the available experimental evidence obtained with insects speaks strongly in favor of the correlation model as the basis of local-motion detection, ${ }^{25}$ this issue is still 
controversial with respect to humans. ${ }^{7}$ Most experimental evidence in favor of a quadratic nonlinearity as the decisive constituent of biological motion-detection systems, and thus in favor of the correlation model, has been derived from time-averaged responses. For these conditions several predictions can be made that can be tested experimentally. (1) Superposition property: the average response to the Fourier components of a stimulus pattern presented simultaneously is simply the sum of the average responses to the individual components. ${ }^{8,16,22,23,26}$ (2) Phase invariance: the mean response of a movement detector of the correlation type does not depend on the relative phases of the spatial Fourier components of a stimulus pattern moved with a constant speed..$^{8,16,22,23,26-30}$ The phase invariance and the superposition property are, of course, closely related to each other. (3) Pattern dependence: the movement-detector output is not determined unambiguously by the stimulus velocity but depends also on the structure of the stimulus pattern. ${ }^{9}$ When the pattern is moved with a constant velocity in one direction and mean responses are taken, the response optima of the movement-detection system depend on the temporal frequency, which is the product of the angular velocity of the pattern and its spatial frequency, rather than on velocity itself. ${ }^{9,23,25}$ This property has often been studied in motion vision of insects. ${ }^{24,25,28,31-34}$ Psychophysical experiments indicate that the performance of the human motion-vision system also depends on both the spatial wavelength of the pattern and its velocity. ${ }^{35-41}$ (4) Contrast dependence: The mean response of the correlator model depends quadratically on pattern contrast. This prediction was tested experimentally in the insect visual system and could be shown to be satisfied at low contrast, whereas at high contrast the response was found to saturate $2,24,42-44$ because of nonlinearities in the movement-detector input channels. ${ }^{44}$

Only recently were the dynamical response properties of correlation type movement detectors used as a distinguishing feature of this motion-detection mechanism. In particular, it was predicted and experimentally verified in direction-selective motion-sensitive visual interneurons in the fly that the time course of the spatially integrated response of an array of movement detectors is proportional to stimulus velocity only within a limited dynamic range. When the velocity changes sufficiently quickly, there are characteristic deviations of the response from proportionality to pattern velocity. ${ }^{44,45}$

Here we present an even more specific criterion that may distinguish a motion-detection scheme with a quadratic nonlinearity, such as a correlation-type movement detector, from other models. This criterion is based on the timedependent performance of local-movement detectors rather than on their temporally or spatially integrated responses. It allows us to characterize formally the nonlinear interaction between the movement-detector input channels. This is done here for the visual system of the fly. As our indicator of the performance of the fly motion-detection system we use the intracellularly recorded responses of a visual interneuron that integrates the signals of large retinotopic arrays of local-movement detectors. To characterize responses of individual movement detectors, spatial integration was prevented by moving the stimulus pattern behind a small vertical slit. This means that, in the case of a vertical sine-wave grating, only a fraction of a spatial wavelength is seen by the animal at any time. This simple technique was applied recently in a behavioral analysis of the motion-detection system of the fly. ${ }^{46}$ Apart from analyzing the responses of individual movement detectors, we also study the consequences of their specific properties for the spatially integrated motion-detector response. This is done by recording from the same cell with the pattern alternately stimulating large parts of the receptive field of the cell and being visible only through the slit.

\section{MATERIAL AND METHODS}

\section{A. Electrophysiology}

\section{Preparation}

The experiments were performed with 1- to 2-day-old female blowflies (Calliphora erythrocephala), which were obtained from laboratory cultures. The animals were anesthetized briefly with carbon dioxide and mounted ventral side up with a mixture of wax and colophonium on a small piece of glass. A small hole was cut in the back of the head capsule to gain access to the brain. The trachea and air sacs that cover the brain areas to be recorded from were dissected away carefully. To avoid movements of the brain caused by peristaltic contractions of the esophagus, the proboscis of the fly was cut away, and the gut was pulled out. This method permitted stable intracellular recordings from large visual interneurons [horizontal (HS) cells] for as long as 45 min. During the experiment the opening in the fly's head capsule was supplied with Ringer solution (for a formula, see Ref. 47).

\section{Recording}

Electrodes were pulled with a Brown-Flaming micropipette puller (P-77) using glass capillaries with a diameter of $1 \mathrm{~mm}$ (Clark, GC100F-10). When filled with $1 M \mathrm{KCl}$, the electrodes had resistances of approximately 30-50 M 2 . The intracellularly recorded graded membrane potential changes of the HS cells were fed to an IBM-AT computer through a 12-bit analog-to-digital converter (Data Translation DT2801-A) at a sampling rate of $2 \mathrm{kHz}$. The programs used for data evaluation were written in ASYST (Keithley Instruments).

\section{Stimulation}

A CRT screen (Tektronix 608) was placed in front of the right eye at an angle of $45^{\circ}$ from the fly's frontal midline. As seen by the fly, the monitor had a horizontal angular extent of $68^{\circ}$ and a vertical extent of $81^{\circ}$. The stimulus pattern was produced by an image synthesizer (Picasso, Innisfree, Inc.), which was controlled by programs written in ASYST on an IBM-AT computer. Three different time-dependent stimulus patterns were used in the experiments: (1) A stimulus pattern with an intensity modulated sinusoidally along its horizontal axis (sine-wave grating) was moved horizontally either in the cell's preferred direction (i.e., from the front to the back) or in the null direction (i.e., from the back to the front). The spatial wavelength of the pattern was either $68^{\circ}$ or $17^{\circ}$. (2) The brightness of a stationary sine-wave grating with a wavelength of $17^{\circ}$ was modulated sinusoidally; this stimulus is referred to hereafter as counterphase flicker. (3) The brightness of a spatially uniform pattern was modulated sinusoidally; this stimulus is referred to hereafter as field 

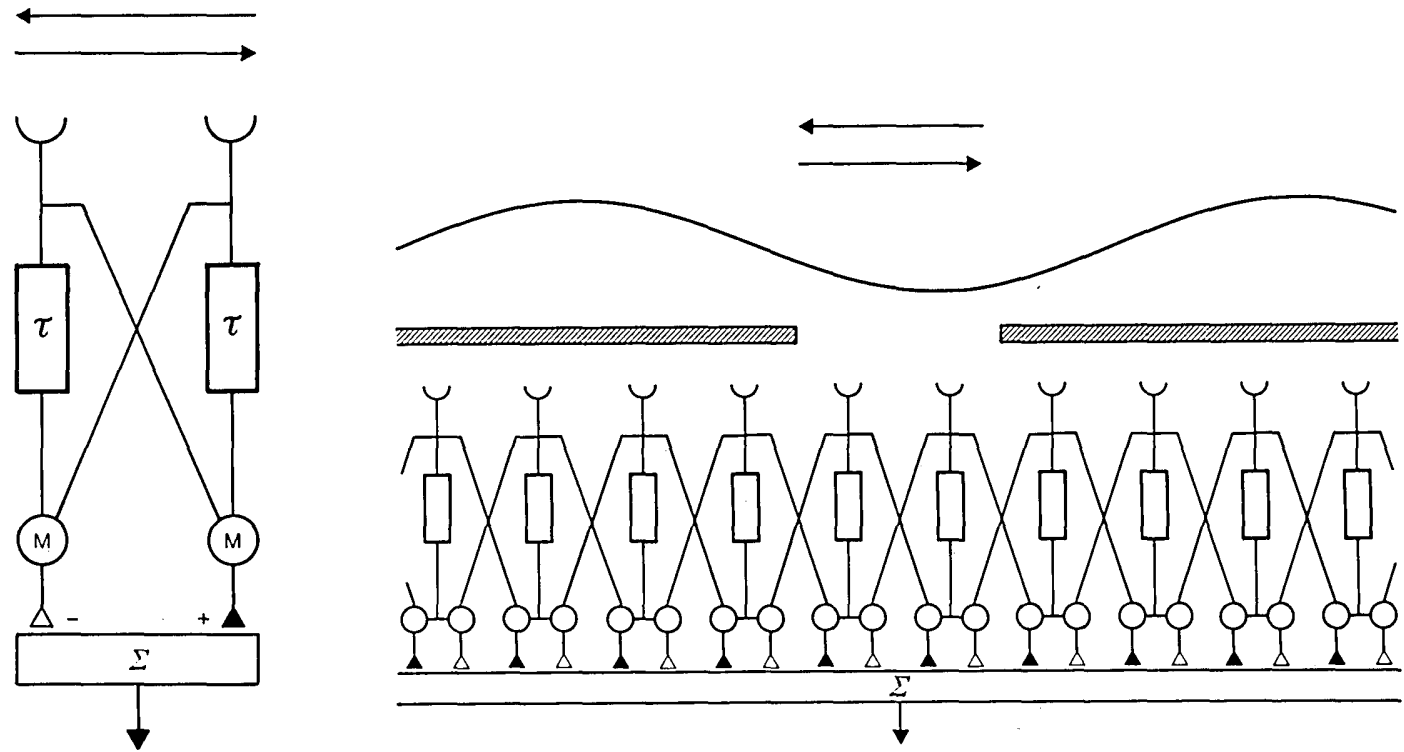

Fig. 1. Schematic representation of (left) an individual movement detector and (right) a one-dimensional array of movement detectors, illustrating the stimulus conditions of some of the experiments shown in this study. Each movement detector consists of two mirrorsymmetrical subunits. In each subunit the signal of one input channel is delayed in some way, such as by a low-pass filter with a time constant $\tau$, and subsequently is multiplied with the undelayed signal of the neighboring input channel. The final detector output is given by the difference of the subunits' outputs. (The open inverse arrowhead indicates the channel that is subtracted.) In the diagram on the right-hand side, an array of movement detectors is integrated by some summating element. In our experiments on the visual system of the fly, this element corresponds to the HS cell. The slit paradigm used in some of our experiments is illustrated schematically. Instead of the entire array, only a single movement detector is exposed to the moving sine-wave pattern. The other detectors are prevented by a stationary mask from contributing to the overall response. In this way, it is possible, at least in principle, to study the properties of individual movement detectors while monitoring the response of a spatially integrating element (for further details see the text).

flicker. The mean luminance of all stimuli was $\sim 25 \mathrm{~cd} / \mathrm{m}^{2}$. The contrast $\left(I_{\max }-I_{\min }\right) /\left(I_{\max }+I_{\min }\right)$, with $I_{\max }$ and $I_{\min }$ denoting the maximum and minimum luminances, respectively, could be varied between $\sim 0.01$ and 0.5 . These patterns were seen by the fly through rectangular windows of two different sizes. The window either comprised the entire monitor screen (and consequently had a size of $68^{\circ} \times 81^{\circ}$ ) or was only a vertical slit with a size of $8.5^{\circ} \times 81^{\circ}$. This slit was generated electronically and located in the middle of the monitor's horizontal axis. When the fly was exposed to the time-dependent stimulus pattern through the slit, only $1 / 8$ of a spatial wavelength (in the case of the $68^{\circ}$ sine-wave grating) could be seen by the fly.

\section{B. Computer Simulations}

A one-dimensional array consisting of a variable number of movement detectors (see Fig. 1) was simulated on an IBMAT computer. The programs were written in ASYST. The relevant details of the model simulations are given in the Results section and the figure captions.

\section{RESPONSE PROPERTIES OF INDIVIDUAL MOVEMENT DETECTORS}

Our evidence in favor of a quadratic nonlinearity as the essential part of the fly's movement-detection system does not rely on temporally or spatially averaged responses. In contrast, it is based on the performance of an individual movement detector in time. For convenience, only steadystate responses are considered here. To make predictions that can be tested experimentally, we had to select a specific representative of the general class of movement detectors with a quadratic nonlinearity. We have chosen the so-called correlation type of movement detector, since this model has proved to be successful in explaining motion vision in insects. ${ }^{2,8,9,20-23,45,48,49}$ Another model scheme, the so-called spatiotemporal energy model, ${ }^{19}$ is, despite considerable differences in its internal structure, at its output mathematically equivalent to the correlation model ${ }^{17,19}$ and thus leads to equivalent predictions.

\section{A. Theoretical Analysis}

A movement detector of the correlation type is illustrated in Fig. 1. It has two input channels, which are spatially separated by the angle $\Delta \varphi$, the so-called sampling base. Each detector is assumed to consist of two mirror-symmetrical subunits that share the same input channels in space. The signal of one branch of each subunit is delayed by passing it through an appropriate linear filter. In each subunit the delayed signal originating from one retinal location is multiplied with the instantaneous signal of the neighboring input channel. The final output of a movement detector is given by the difference between the two subunit outputs. With $A_{1}(t)$ and $A_{2}(t)$ representing the input signals at the right and left movement-detector input channels and $B_{1}(t)$ and $B_{2}(t)$ representing the corresponding filtered signals, one obtains for the output $R(t)$ of a movement detector

$$
R(t)=A_{2}(t) B_{1}(t)-A_{1}(t) B_{2}(t) .
$$

As formulated here, the movement detector is perfectly antisymmetrical; i.e., it responds with the same amplitude, but with opposite signs, to motion in its preferred and null directions, respectively. This, however, is true only as long as the corresponding operations in the two detector subunits 
are exactly identical. Small differences may alter the final movement-detector output qualitatively. Formally, there are two possible sites in the model that may be liable to imperfections. Either slightly different properties of the temporal filters or imperfections in the subtraction stage may unbalance the two detector subunits. Since a perfectly balanced movement detector is unlikely to be realized in biological systems, we derived our model predictions from a more general formulation of the movement-detector output that allowed for imperfections in both the temporal filters and the subtraction stage.

As specific input signals, we used grating patterns that are sinusoidally modulated along their direction of motion. This allows us to treat the problem in only one spatial dimension. Assume that we use a sine-wave grating with a spatial wavelength $\lambda$ that moves with a constant angular velocity $v$ from right to left (the detector's preferred direction as referred to by the superscript $\mathbf{p})$. With $v / \lambda$ corresponding to the temporal frequency (in hertz) and $\omega=2 \pi(v /$ $\lambda)$ corresponding to the circular frequency (in radians per second), and $\varphi$ being the phase angle of the pattern at time $t$ $=0$, one obtains, for the input signal of the left movementdetector channel,

$$
A_{1}{ }^{\mathrm{p}}(t)=I+\Delta I \sin (\omega t+\varphi) .
$$

With $2 \pi \Delta \varphi / \lambda$ corresponding to the phase difference between the two detector input signals, one accordingly obtains for the right input signal

$$
A_{2}{ }^{\mathrm{p}}(t)=I+\Delta I \sin (\omega t+\varphi-2 \pi \Delta \varphi / \lambda) \text {. }
$$

In Eqs. (2a) and (3a) $I$ and $\Delta I$ refer to the representation of the mean luminance of the stimulus pattern at the movement detector input and to the modulation, respectively. It should be emphasized that $I$ does not necessarily correspond to the mean luminance of the stimulus pattern as received by the eye. In the case of the fly's motion-detection system, for instance, there is good evidence that the mean luminance has been eliminated to a high degree from the movementdetector input signals. ${ }^{44}$ When the pattern moves in the opposite direction (the detector's null direction, as indicated by the superscript $n$ ), one obtains, for the movement-detector input functions,

$$
A_{1}{ }^{\mathrm{n}}(t)=I+\Delta I \sin (-\omega t+\varphi)
$$

and

$$
A_{2}{ }^{\mathrm{n}}(t)=I+\Delta I \sin (-\omega t+\varphi-2 \pi \Delta \varphi / \lambda) \text {. }
$$

Having passed the movement-detector filters, $A_{1} \mathrm{p}(t)$ and $A_{2}{ }^{\mathrm{p}}(t)$ transform to

$$
B_{1}{ }^{\mathrm{n}}(t)=I+F_{1}(\omega) \Delta I \sin \left[-\omega t+\varphi-\phi_{1}(\omega)\right]
$$

and

$B_{2}^{\mathrm{n}}(t)=I+F_{2}(\omega) \Delta I \sin \left[-\omega t+\varphi-\phi_{2}(\omega)-2 \pi \Delta \varphi / \lambda\right]$.

$F_{1}(\omega), F_{2}(\omega)$ and $\phi_{1}(\omega), \phi_{2}(\omega)$ denote the amplitude-frequency and phase-frequency functions of the filters in the left and right detector input channels. For convenience the filters are assumed to represent low-pass filters, implying that $F_{1}(\omega)=1, F_{2}(\omega)=1, \phi_{1}(\omega)=0$, and $\phi_{2}(\omega)=0$, for $\omega=0$. The final movement-detector responses can be obtained by inserting Eqs. (2)-(5) into Eq. (1a). To allow for imperfections in the subtraction process Eq. (1a) is modified slightly by weighting one of the detector subunits by a factor $g$, which, in a first approximation, is assumed to be constant and independent of the stimulus conditions:

$$
R(t)=A_{2}(t) B_{1}(t)-g A_{1}(t) B_{2}(t) .
$$

The responses to motion in both the preferred $\left(R^{p}\right)$ and the null $\left(R^{\mathrm{n}}\right)$ directions as derived from Eqs. (1b)-(5) are composed of three components. One of them is independent of time, and the two others vary as a function of time. Of the latter two components the first is modulated with the fundamental harmonic of the periodic stimulus, and the second one depends on the second harmonic. The response to motion in the preferred direction then reads as

$$
R^{\mathrm{p}}(t)=S_{c}^{\mathrm{p}}+S_{\omega t}^{\mathrm{p}} \sin \left(\omega t+\psi_{\omega t}^{\mathrm{p}}\right)+S_{2 \omega t}^{\mathrm{p}} \sin \left(2 \omega t+\psi_{2 \omega t}^{\mathrm{p}}\right)
$$

with the amplitude factors and phase angles amounting to

$$
\begin{aligned}
S_{c}^{\mathrm{p}}= & I^{2}(1-g)+\left(1 / 2 \Delta I^{2}\left\{F_{1}(\omega) \cos \left[\phi_{1}(\omega)+2 \pi \Delta \varphi / \lambda\right]\right.\right. \\
& \left.-g F_{2}(\omega) \cos \left[\phi_{2}(\omega)-2 \pi \Delta \varphi / \lambda\right]\right\}, \\
S_{\omega t}^{\mathrm{p}}= & I \Delta I\left[\left(O^{\mathrm{p}}\right)^{2}+\left(P^{\mathrm{p}}\right)^{2}\right]^{1 / 2}, \\
\psi_{\omega t}^{\mathrm{p}}= & \arctan \left(O^{\mathrm{p}} / P^{\mathrm{p}}\right),
\end{aligned}
$$

with

$$
\begin{aligned}
O^{\mathrm{p}}= & \sin (\varphi-2 \pi \Delta \varphi / \lambda)+F_{1}(\omega) \sin \left[\varphi+\phi_{1}(\omega)\right] \\
& -g F_{2}(\omega) \sin \left[\varphi+\phi_{2}(\omega)-2 \pi \Delta \varphi / \lambda\right]-g \sin (\varphi), \\
P^{\mathrm{p}}= & \cos (\varphi-2 \pi \Delta \varphi / \lambda)+F_{1}(\omega) \cos \left[\varphi+\phi_{1}(\omega)\right] \\
& -g F_{2}(\omega) \cos \left[\varphi+\phi_{2}(\omega)-2 \pi \Delta \varphi / \lambda\right]-g \cos (\varphi), \\
S_{2 \omega t}^{\mathrm{p}}= & (1 / 2) \Delta I^{2}\left\{F_{1}(\omega)^{2}+g^{2} F_{2}(\omega)^{2}\right. \\
& \left.-2 g F_{1}(\omega) F_{2}(\omega) \cos \left[\phi_{1}(\omega)-\phi_{2}(\omega)\right]\right\}^{1 / 2},
\end{aligned}
$$

$$
\psi_{2 \omega t}^{\mathrm{p}}=\arctan \frac{g F_{2}(\omega) \cos \left[2 \varphi+\phi_{2}(\omega)-2 \pi \Delta \varphi / \lambda\right]-F_{1}(\omega) \cos \left[2 \varphi+\phi_{1}(\omega)-2 \pi \Delta \varphi / \lambda\right]}{F_{1}(\omega) \sin \left[2 \varphi+\phi_{1}(\omega)-2 \pi \Delta \varphi / \lambda\right]-g F_{2}(\omega) \sin \left[2 \varphi+\phi_{2}(\omega)-2 \pi \Delta \varphi / \lambda\right]} .
$$

and

$$
B_{2}^{\mathrm{p}}(t)=I+F_{2}(\omega) \Delta I \sin \left[\omega t+\varphi+\phi_{2}(\omega)-2 \pi \Delta \varphi / \lambda\right]
$$

respectively. Accordingly, $A_{1}{ }^{\mathrm{n}}$ and $A_{2}{ }^{\mathrm{n}}$ transform to
Accordingly, one obtains, for the response of an individual movement detector to motion in its null direction,

$$
R^{\mathrm{n}}(t)=S_{c}{ }^{\mathrm{n}}+S_{\omega t}{ }^{\mathrm{n}} \sin \left(\omega t+\psi_{\omega t}^{\mathrm{n}}\right)+S_{2 \omega t}{ }^{\mathrm{n}} \sin \left(2 \omega t+\psi_{2 \omega t}{ }^{\mathrm{n}}\right),
$$

with 


$$
\begin{aligned}
S_{c}{ }^{\mathrm{n}}= & I^{2}(1-g)+\left(1 / 2 \Delta I^{2}\right)\left\{F_{1}(\omega) \cos \left[\phi_{1}(\omega)-2 \pi \Delta \varphi / \lambda\right]\right. \\
& \left.-g F_{2}(\omega) \cos \left[\phi_{2}(\omega)+2 \pi \Delta \varphi / \lambda\right]\right\}, \\
S_{\omega t}{ }^{\mathrm{n}}= & I \Delta I\left[\left(O^{\mathrm{n}}\right)^{2}+\left(P^{\mathrm{n}}\right)^{2}\right]^{1 / 2}, \\
\psi_{\omega t}{ }^{\mathrm{n}}= & \arctan \left(O^{\mathrm{n}} / P^{\mathrm{n}}\right), \\
O^{\mathrm{n}}= & \sin (\varphi-2 \pi \Delta \varphi / \lambda)+F_{1}(\omega) \sin \left[\varphi-\phi_{1}(\omega)\right] \\
& -g F_{2}(\omega) \sin \left[\varphi-\phi_{2}(\omega)-2 \pi \Delta \varphi / \lambda\right]-g \sin (\varphi), \\
P^{\mathrm{n}}= & -\left\{\cos (\varphi-2 \pi \Delta \varphi / \lambda)+F_{1}(\omega) \cos \left[\varphi-\phi_{1}(\omega)\right]\right. \\
& \left.-g F_{2}(\omega) \cos \left[\varphi-\phi_{2}(\omega)-2 \pi \Delta \varphi / \lambda\right]-g \cos (\varphi)\right\},
\end{aligned}
$$

$$
\begin{aligned}
S_{2 \omega t}{ }^{\mathrm{n}}= & (1 / 2) \Delta I^{2}\left\{F_{1}(\omega)^{2}+g^{2} F_{2}(\omega)^{2}\right. \\
& \left.-2 g F_{1}(\omega) F_{2}(\omega) \cos \left[\phi_{1}(\omega)-\phi_{2}(\omega)\right]\right\}^{1 / 2},
\end{aligned}
$$

$$
\begin{aligned}
S_{c}^{\mathrm{ff}}= & I^{2}(1-g)+\left(1 / 2 \Delta I^{2}\right)\left\{F_{1}(\omega) \cos \left[\phi_{1}(\omega)\right]\right. \\
& \left.-g F_{2}(\omega) \cos \left[\phi_{2}(\omega)\right]\right\}, \\
S_{\omega t}^{\mathrm{ff}}= & I \Delta I\left[\left(O^{\mathrm{ff}}\right)^{2}+\left(P^{\mathrm{ff}}\right)^{2}\right]^{1 / 2}, \\
\psi_{\omega t}^{\mathrm{ff}}= & \arctan \left(O^{\mathrm{ff}} / P^{\mathrm{ff}}\right),
\end{aligned}
$$

with

$$
\begin{aligned}
O^{\mathrm{ff}}= & \left.\sin (\varphi)+F_{1}(\omega) \sin \left[\varphi+\phi_{1}(\omega)\right)\right] \\
& -g F_{2}(\omega) \sin \left[\varphi+\phi_{2}(\omega)\right]-g \sin (\varphi), \\
P^{\mathrm{ff}}= & \cos (\varphi)+F_{1}(\omega) \cos \left[\varphi+\phi_{1}(\omega)\right] \\
& -g F_{2}(\omega) \cos \left[\varphi+\phi_{2}(\omega)\right]-g \cos (\varphi), \\
S_{2 \omega t}^{\mathrm{ff}}= & (1 / 2) \Delta I^{2}\left\{F_{1}(\omega)^{2}+g^{2} F_{2}(\omega)^{2}\right. \\
& \left.-2 g F_{1}(\omega) F_{2}(\omega) \cos \left[\phi_{1}(\omega)-\phi_{2}(\omega)\right]\right\}^{1 / 2},
\end{aligned}
$$

$\psi_{2 \omega t}{ }^{\mathrm{n}}=\arctan \frac{g F_{2}(\omega) \cos \left[2 \varphi-\phi_{2}(\omega)-2 \pi \Delta \varphi / \lambda\right]-F_{1}(\omega) \cos \left[2 \varphi-\phi_{1}(\omega)-2 \pi \Delta \varphi / \lambda\right]}{-F_{1}(\omega) \sin \left[2 \varphi-\phi_{1}(\omega)-2 \pi \Delta \varphi / \lambda\right]+g F_{2}(\omega) \sin \left[2 \varphi-\phi_{2}(\omega)-2 \pi \Delta \varphi / \lambda\right]}$.

It follows from Eqs. (6)-(13) that the amplitudes and phase angles of the different frequency components depend in a characteristic way on (1) the different parameters of the stimulus, such as its spatial wavelength and degree of modulation; (2) the geometrical properties of the eye, such as the angular separation of the two detector input channels; and (3) the characteristics of the movement detector, such as the time constant of the temporal filters. So far it has been assumed that the outputs of the two movement-detector subunits are not perfectly balanced at the subtraction stage; i.e., $g \neq 1, F_{1}(\omega) \neq F_{2}(\omega)$, and $\phi_{1}(\omega) \neq \phi_{2}(\omega)$. Under the special condition of a balanced subunit output, i.e., $g=1$, $F_{1}(\omega)=F_{2}(\omega)$, and $\phi_{1}(\omega)=\phi_{2}(\omega)$, the second-harmonic component of the time-dependent movement-detector response disappears [see Eqs. (12a) and (12b)], leaving only the fundamental frequency component.

A movement detector that is not exactly balanced with respect to the outputs of its constituent subunits becomes sensitive also to temporal modulations of the brightness of a stationary pattern. In case of a spatially homogeneous pattern that is sinusoidally modulated in time (field flicker, as is indicated by the superscript $\mathrm{ff}$ ) both movement-detector input channels receive the same intensity modulations:

$$
A_{1}^{\mathrm{ff}}(t)=A_{2}^{\mathrm{ff}}(t)=I+\Delta I \sin (\omega t+\varphi)
$$

with $\varphi$ corresponding to the phase angle at time $t=0$. Having passed the movement-detector filter, $A^{\text {ff }}$ transforms to

$$
\begin{aligned}
& B_{1}{ }^{\mathrm{ff}}(t)=I+F_{1}(\omega) \Delta I \sin \left[\omega t+\varphi+\phi_{1}(\omega)\right] . \\
& B_{2}{ }^{\mathrm{ff}}(t)=I+F_{2}(\omega) \Delta I \sin \left[\omega t+\varphi+\phi_{2}(\omega)\right] .
\end{aligned}
$$

Again, $F_{1}(\omega), F_{2}(\omega), \phi_{1}(\omega), \phi_{2}(\omega)$ correspond to the amplitude- and phase-frequency functions of the movement-detector filters. One finally obtains, for the response of an individual movement detector to field flicker stimulation,

$$
R^{\mathrm{ff}}=S_{c}^{\mathrm{ff}}+S_{\omega t}^{\mathrm{ff}} \sin \left(\omega t+\phi_{\omega t}^{\mathrm{ff}}\right)+S_{2 \omega t} \underset{ }{\mathrm{ff}} \sin \left(2 \omega t+\psi_{2 \omega t} \underset{\mathrm{ff}}{\mathrm{ff}}\right),
$$

with the amplitude factors and phase angles defined by

$$
\psi_{2 \omega t}^{\mathrm{ff}}=\arctan \frac{g F_{2}(\omega) \cos \left[2 \varphi+\phi_{2}(\omega)\right]-F_{1} \cos \left[2 \varphi+\phi_{1}(\omega)\right]}{F_{1}(\omega) \sin \left[2 \varphi+\phi_{1}(\omega)\right]-g F_{2} \sin \left[2 \varphi+\phi_{2}(\omega)\right]} .
$$

As do the responses to motion, the response to field flicker stimulations consists of three components. One of them is independent of time, one is modulated with the fundamental frequency, and one is modulated with the second harmonic of the periodic stimulus.

As is obvious from the above theoretical formulations of the movement-detector output, its time course depends on a number of parameters. In a biological system most of them cannot easily be specified experimentally. Nevertheless, it is possible to make predictions that are independent of these parameters and depend on only the assumption of a multiplicationlike interaction as the essential nonlinearity of the movement detector. In this case, only the fundamental and second harmonics of the temporal frequency of the stimulus are found in the time course of the movement-detector responses. Of course, even in the case of a multiplicative interaction this is true only if the sinusoidally modulated retinal input signals are not distorted significantly by any nonlinearity in the input channels to the multiplication or at the detector output. The occurrence of only the first and second harmonics in the response of a movement-detection system is compatible with this type of multiplicative interaction and, additionally, suggests the absence of significant distortions of its input and output signals.

One can go a step further and put forward a quantitative prediction. As can be derived from Eqs. (12) and (23), the powers $P_{2 \omega t}$ of the second-harmonic components (i.e., the squared amplitudes of these response frequency components) of the responses to motion in the preferred and null directions and to field flicker stimulation are the same:

$$
\begin{aligned}
P_{2 \omega t}^{\mathrm{p}}= & P_{2 \omega t}^{\mathrm{n}}=P_{2 \omega t}^{\mathrm{ff}}=(1 / 4) \Delta I^{4}\left\{F_{1}(\omega)^{2}+g^{2} F_{2}(\omega)^{2}\right. \\
& \left.-2 g F_{1}(\omega) F_{2}(\omega) \cos \left[\phi_{1}(\omega)-\phi_{2}(\omega)\right]\right\} .
\end{aligned}
$$

In contrast, the power $P_{\omega t}$ of the fundamental frequency is expected to vary greatly with the stimulus conditions [see Eqs. (8), (10), (11), (19), (21), and (22)]. Formally, the filters 
in the two movement-detector subunits and the exact properties of the subtraction stage (as characterized by the parameter g) determine which stimulus results in the most pronounced fundamental frequency response component. It should be emphasized that this result is not predicted by a special version of the movement detector proposed by Kirschfeld ${ }^{12}$ in which the subtraction stage is omitted and the two detector input channels contain a low-pass filter and a high-pass filter of the same order. This model becomes sensitive to flicker stimulation, for instance, when different time constants are assumed for its two temporal filters. However, even then, the power of the fundamental frequency component should be the same for all three stimulus conditions. Moreover, it should be mentioned that Grzywacz and $\mathrm{Koch}^{26}$ derived a criterion for a quadratic nonlinearity, the frequency doubling index, which is somewhat related to Eq. (25). Whereas a doubling index of 1 represents evidence for a truly second-order system, smaller values are assumed to indicate deviations from this type of nonlinearity. Since in the case of a balanced movement detector the frequency doubling index is zero, this measure for a quadratic nonlinearity of the movement-detection system could not be used in the present context.

Another qualitative prediction is concerned with the contrast dependence of the different frequency components of the movement-detector response. The relative contribution of the second harmonic in the responses should decrease with decreasing contrast, leaving the fundamental frequency as the dominant component in the response profiles at low contrast. This is because the fundamental frequency depends linearly on pattern contrast, while the second harmonic depends on it in a quadratic way [compare Eqs. (8) and (12) as well as Eqs. (19) and (23)].

All these predictions are independent of the specific type of movement-detector filter. Instead, they are based on the assumption of a multiplicative interaction as the essential nonlinearity inherent in this type of movement-detection scheme. To be exact, however, they also depend on the assumption that at the subtraction stage both detector subunits contribute to the final movement-detector response with a constant gain; i.e., the weighting factor $g$ is constant. Despite this qualification, a particular movement-detection system can be represented by a correlation-type movement detector if these predictions are satisfied.

\section{B. Responses of the Horizontal}

In our experiments the responses of the direction-selective, motion-sensitive HS cell in the fly's third visual ganglion ${ }^{47}$ were used as an indicator of the performance of the movement-detection system. This cell is advantageous for an investigation of a biological motion-detection system from an analytical point of view. It can be identified individually in each animal by physiological and anatomical criteria. Its main response mode is that of graded membrane potential changes rather than spike activity. Intracellular recording from this cell thus allows us to monitor the summated synaptic potentials of its presynaptic elements. Since these presynaptic elements are assumed to represent the local-movement detectors (for a detailed discussion, see Ref. 50), the computations underlying motion detection can be analyzed without much interference from other processes.

The HS cell spatially pools the output of large retinotopic arrays of horizontally oriented movement detectors. There- fore the response of an individual movement detector can be analyzed only if spatial integration is prevented in some way. This was done here by a technique that was used previously in experiments in which behavioral responses were taken as an indicator of the performance of the movement-detection system. ${ }^{46}$ With this technique a large number of movement detectors are stimulated synchronously, instead of only a single one. In a first approximation, this can be achieved by presenting the stimulus pattern to the eye only through a vertical slit. If the brightness of the pattern is modulated along its horizontal axis and the slit is relatively small as compared with the spatial wavelength of the pattern, the corresponding input channels of different movement detectors being distributed along the vertical extent of the slit get virtually the same input signals. A larger pattern wavelength thus allows one to use a wider slit and consequently to get a larger number of almost synchronously stimulated movement detectors contributing to the cell's response. On the other hand, if the spatial wavelength of the pattern is too large, the phase difference between the two input channels of a given movement detector, and, as a consequence, its response, will become too small. A slit width of $1 / 8$ spatial wavelength, as is used here, was found to be a good compromise in this respect.

With this slit paradigm the responses of the HS cell were tested under three different stimulus conditions: (1) The grating was moved from the front to the back, in the cell's preferred direction. (2) It was moved from the back to the front, in the cell's null direction. (3) The brightness of the slit was spatially homogeneous but modulated sinusoidally in time. The responses of HS cells to these stimulus conditions as averaged for nine different flies are shown in Fig. 2.

The responses to motion in the cell's preferred and null directions are not mirror symmetrical (upper diagrams in Fig. 2). They do not have the same time course with only the sign of the response being inverted. This suggests that, in the context of the movement-detection scheme as explained above, the movement detector is not perfectly balanced. This suggestion is supported further by the observation that the cell shows a pronounced response to flicker stimulation. The responses are periodic under all stimulus conditions used in the experiment, with a strong frequency component corresponding to the temporal frequency of the stimulus. However, higher-frequency components are visible. On the average the responses are different from zero. They are positive and negative with respect to the cell's resting potential for motion in the preferred and null directions, respectively. The average response to field flicker stimulation is also positive.

To compare the experimental data with the theoretical predictions the contribution of the different frequency components to the different responses were determined. The mean power spectra of the responses of the different flies are shown in Fig. 2 below the corresponding time-dependent diagrams. For all stimulus conditions the fundamental frequency and the second harmonic are predominant. The contributions of higher harmonics to the total response are only approximately $7 \%, 15 \%$, and $11 \%$ for motion in the preferred direction, motion in the null direction, and flicker stimulation, respectively. These findings present good evidence in favor of a multiplicative interaction as the essential nonlinearity underlying motion detection in the fly's visual system. Moreover, these findings suggest that the sinusoi- 

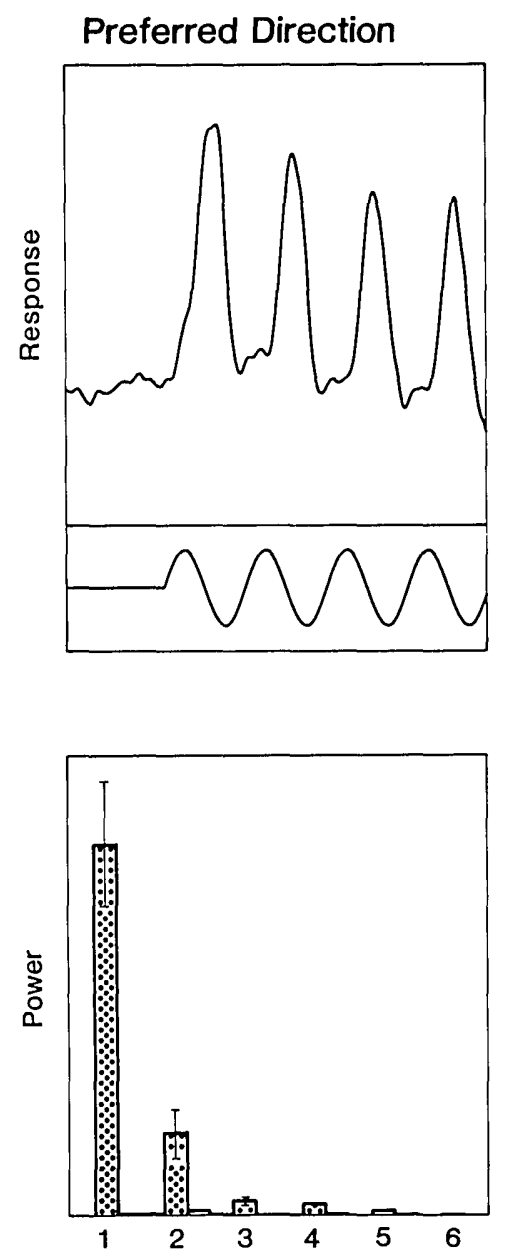

Null Direction

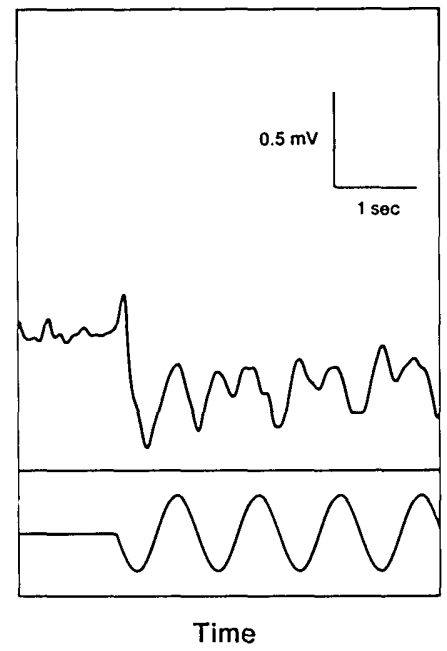

Field Flicker
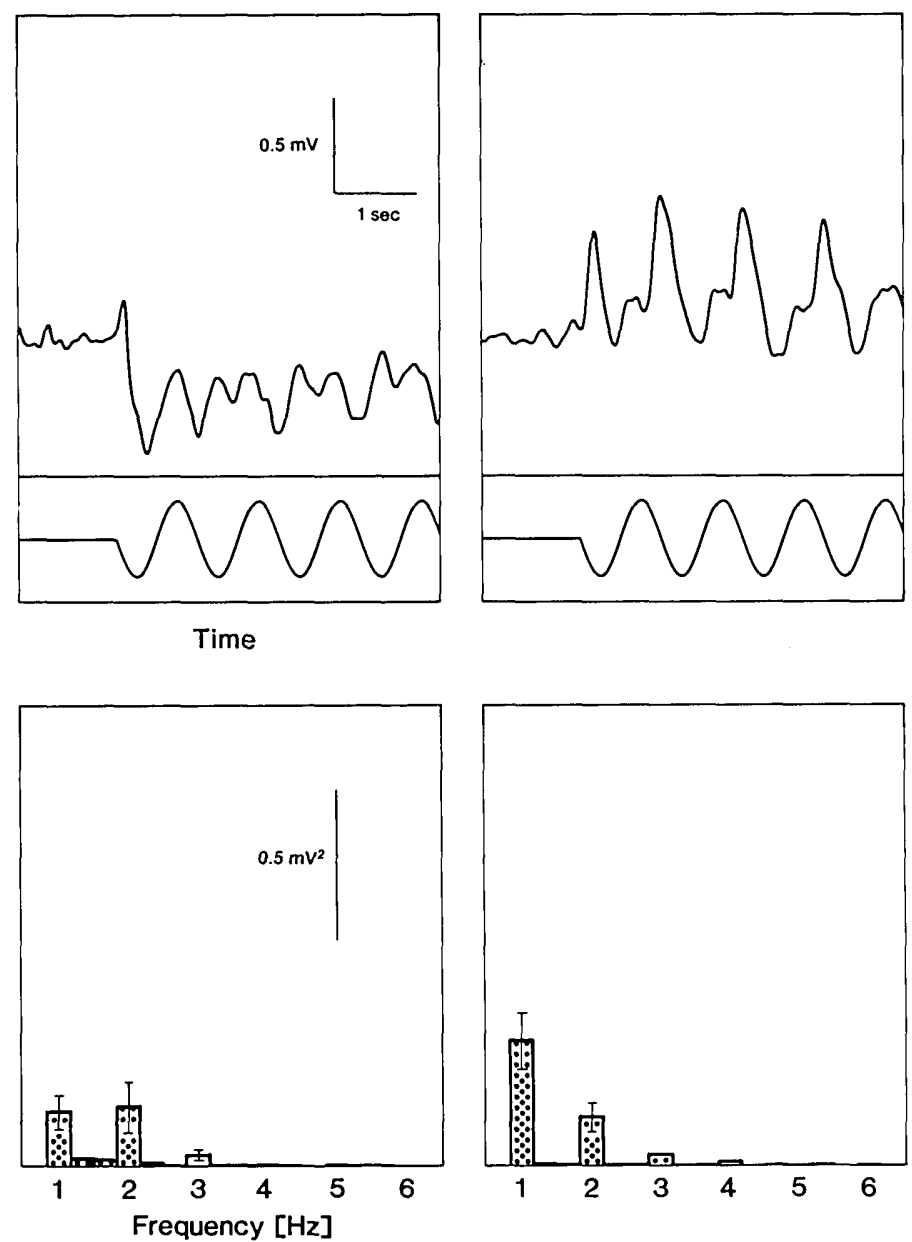

Fig. 2. Responses of movement detectors to motion and flicker stimulation. The responses were recorded intracellularly from a HS cell in the third visual ganglion of the fly's brain. The fly was exposed to the time-dependent stimulus pattern only through a vertical slit that had an angular width of $8.5^{\circ}$. Three different stimulus conditions were used: (Left) A vertical sine-wave grating with a spatial wavelength of $68^{\circ}$ moved in the cell's preferred direction, i.e., from the front to the back. (Middle) The same grating moved in the cell's null direction, i.e., from the back to the front. (Right) The slit appeared spatially homogeneous, but its brightness was modulated sinusoidally in time (field flicker stimulation). The temporal frequency was $1 \mathrm{~Hz}$ in all cases. The upper diagrams show the time-dependent response traces, each averaged from the same sample of nine flies and a total of 113 stimulus presentations. The time course of the brightness at the middle of the slit is displayed below each time-dependent plot. The bottom diagrams show the power spectra of the time-dependent responses. These spectra were computed from the three last periods of the responses by using a fast-Fourier-transform algorithm. They represent the mean values and the standard error of the mean. The first response cycle was omitted to eliminate the effects of potential response transients caused by the onset of stimulus motion. Irrespective of the stimulus conditions, essentially the fundamental frequency and the second harmonic of the temporal frequency of the stimulus contribute to the responses. Whereas the contribution of the second harmonic is relatively constant, the fundamental frequency varies under the different stimulus conditions.

dal profiles of the detector input functions are not distorted much by any nonlinearity preceding the multiplication stage, since higher harmonics would then be expected to contribute considerably to the final detector response. The conclusion that a correlation-type movement detector underlies motion vision in the fly is further supported by the fact that the contribution of the second harmonic to the total response is almost the same for all these stimulus conditions. In contrast, the fundamental frequency component differs considerably under the different stimulus conditions. Hence the relationship among the different frequency components as obtained under the different stimulus conditions is in excellent agreement with the theoretical predictions of Section 2. These findings, however, can hardly be reconciled with the model version proposed by Kirschfeld (see above). ${ }^{12}$
A further critical prediction pertained to the contrast dependence of the second harmonic (see Subsection 3.A). This was tested experimentally in two flies. Since the relative contribution of the second harmonic was most pronounced in the response to motion in the cell's null direction, the results obtained under this stimulus condition were chosen for demonstration in Fig. 3. Under the high-contrast condition the contrast amounted to 0.30 . In accordance with the results shown in Fig. 2, there is a conspicious component corresponding to the second harmonic of the temporal frequency in the power spectrum of the response. At low contrasts ( $\sim 0.03$ in Fig. 3$)$ the response amplitude decreases considerably. It is most striking, however, that the response profiles alter their shapes considerably. As is revealed by the corresponding power spectrum, the fundamental frequency is almost the only frequency component in the re- 
sponse at low contrast, which is quite in accordance with the theoretical expectation.

These experimental findings allow us to draw three principal conclusions with respect to the organization of the localmovement detectors in the fly's visual system: (1) The essential nonlinear interaction between input signals from neighboring points in visual space can be described well by a multiplication. This conclusion holds not only for contrasts near threshold but for contrasts of as much as 0.30 . This finding severely constrains possible cellular models that perform the multiplication in neuronal terms (see Section 5). (2) At least for contrasts as high as those used here, the time course of the detector input signals is not distorted much before it is multiplied with the signal originating from the neighboring input channel. This conclusion significantly constrains the possible processing of the retinal input signals before movement detection. (3) The movement detector is not perfectly antisymmetrical. This suggests, in terms of the model analyzed in Section 2, that the two detector subunits are not perfectly balanced, because of slightly different properties of the temporal filters or of imperfections in the subtraction stage. In the latter case, the gain of the two detector subunits can be concluded to be essentially independent of the stimulus conditions and the movement detector's response amplitude. Since the average response to field flicker stimulation is always positive, it can be concluded further that the subunit that is subtracted always has the smaller gain and also that this asymmetry cannot be due to random fluctuations of some variables in the system. Again, this conclusion will play an important role in establishing cellular models of the movement detector.

\section{Computer Simulation of the Movement-Detector Response}

To test further the conclusion that the characteristic time courses of the single movement-detector responses can be explained by the correlation type of movement detector as explained in Subsection 3.A, the responses were simulated on the basis of this model. To do this, the different model parameters had to be specified appropriately. Of course,

\section{Low Contrast}
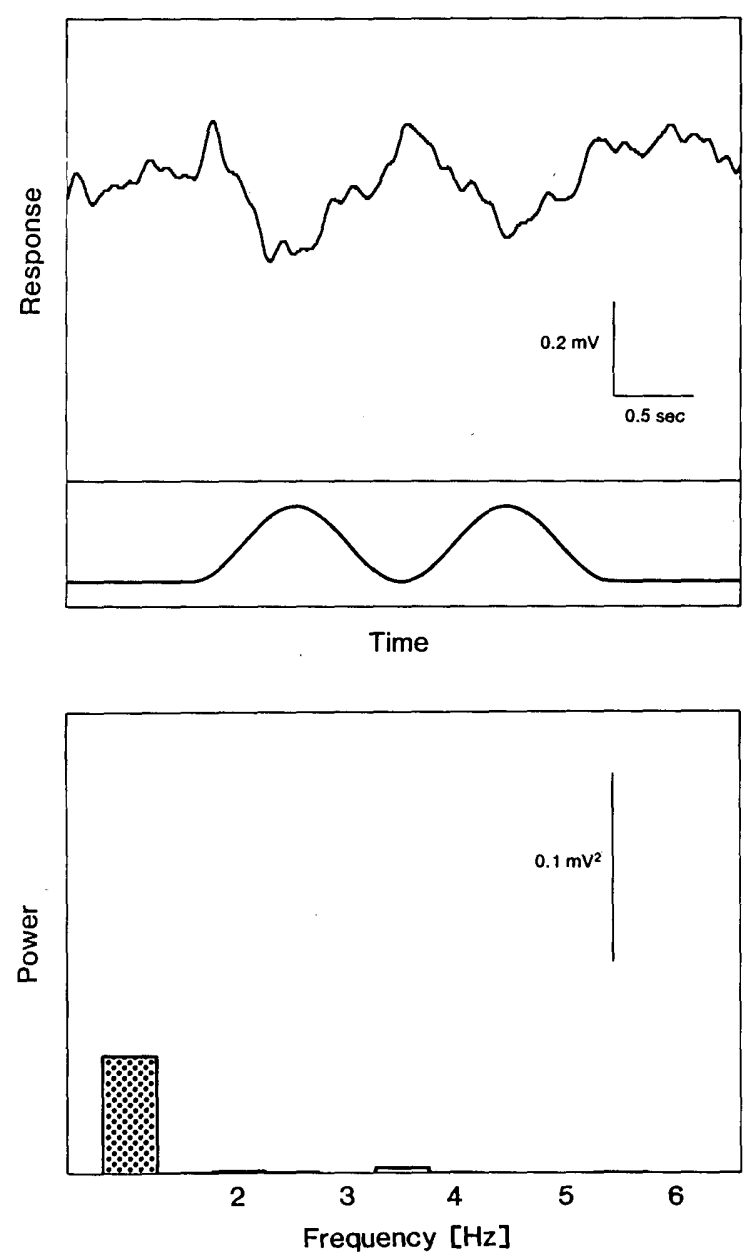

High Contrast
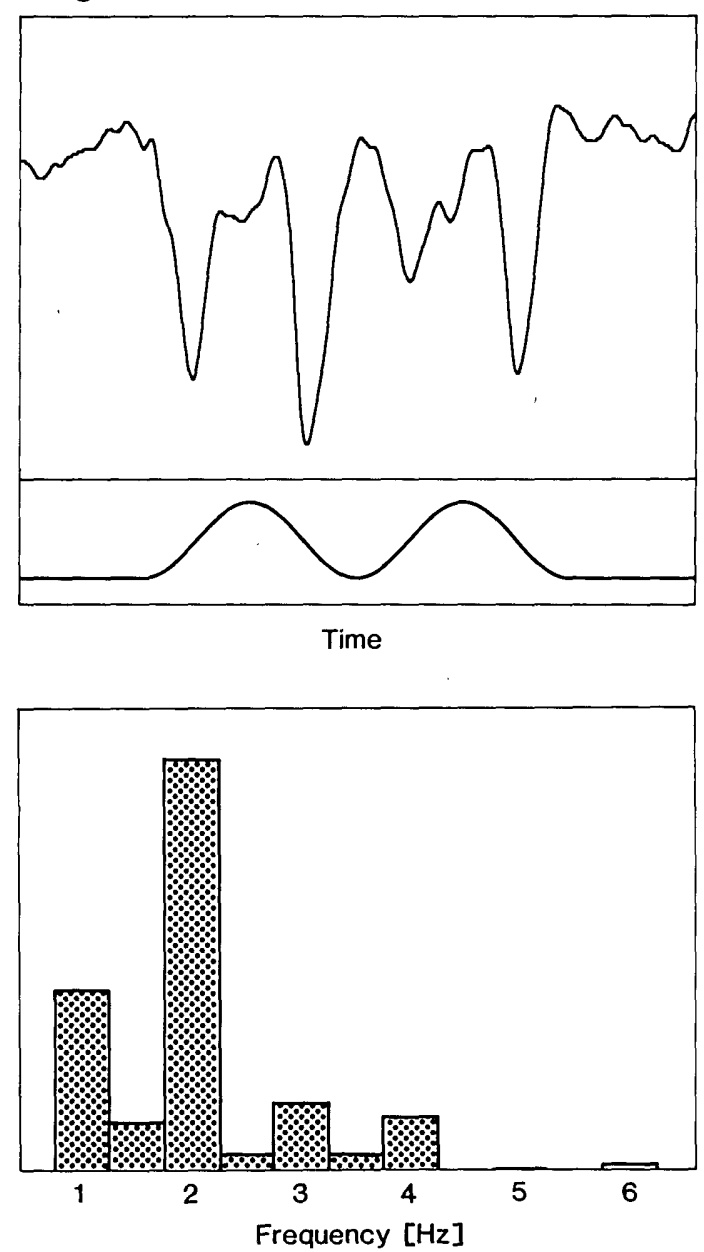

Fig. 3. Responses of movement detectors to motion at low and high pattern contrasts. The responses are averages of two HS cells and a total of 40 stimulus presentations. They were obtained by using the slit paradigm with the pattern moving for two cycles in the cell's null direction. The stimulus conditions were the same as described for the middle diagrams of Fig. 2, except that the contrast amounted to (left) 0.03 and (right) 0.30 . The stimulus traces are shown below the time-dependent plots. The bottom diagrams show the power spectra that were computed from the two cycles of the time-dependent responses. The relative contributions of the fundamental frequency and second harmonic of the temporal frequency of the stimulus depend on the contrast of the pattern. At low contrasts, the fundamental harmonic predominates, while at higher contrasts there is a strong contribution also of the second harmonic. 


\section{Preferred Direction}

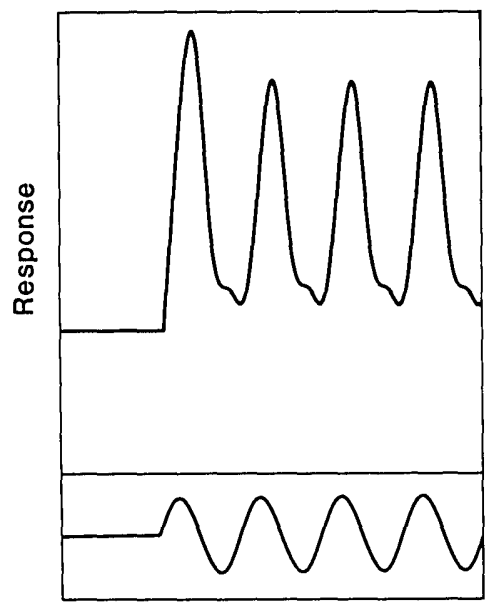

Null Direction

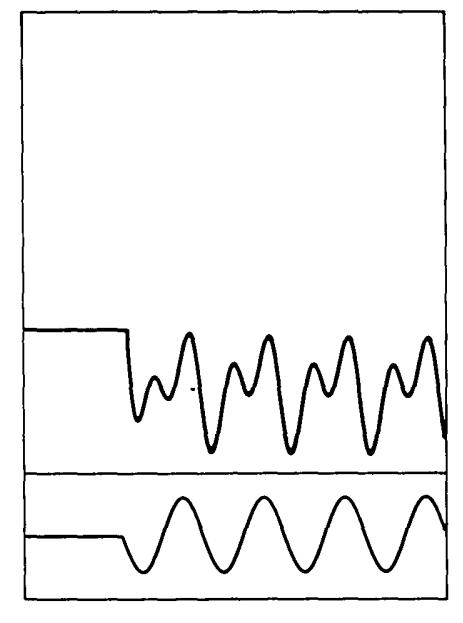

Field Flicker

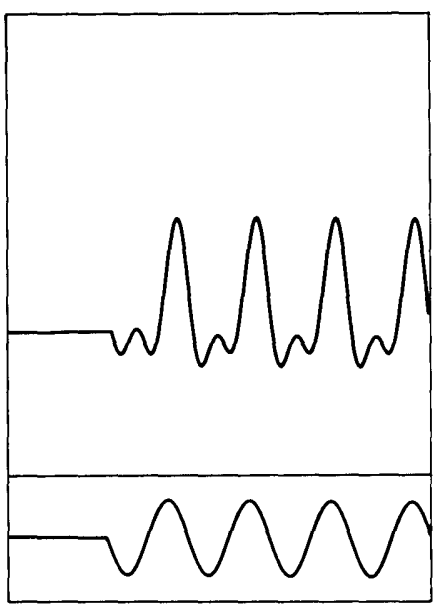

Fig. 4. Computer simulation of the response of a movement detector of the correlation type to motion and flicker stimulation. In both detector subunits a first-order low-pass filter with a time constant of $100 \mathrm{msec}$ was used as the movement-detector filter. The background luminance was subtracted to a high degree from the input signal, leading to an effective contrast of 2.8 at the movement-detector input. The relative contributions of the different frequency components were adjusted approximately to the values obtained in the experiments shown in Fig. 2 by varying the gain of the detector subunit that is subtracted. In the simulations shown here $g$ was 0.89 . The stimulus conditions were essentially the same as in the corresponding experiments shown in Fig. 2. A sine-wave grating was moved in either (left) the preferred direction or (middle) the null direction of the movement detector. The pattern wavelength was then 36 times the angular distance between the two movement-detector input channels. This was of the same order of magnitude as in the corresponding experiments shown in Fig. 2. In the diagram on the right, both input channels received the same input, which was modulated sinusoidally in time. In all cases, the temporal frequency was $1 \mathrm{~Hz}$. The traces below the detector responses give the brightness modulation of the movement-detector input. The computed responses show that the time course of the experimentally determined results of Fig. 2 can be accounted for on the basis of the movement-detector model.

this is to a high degree arbitrary, although the most important parameters can be estimated on an empirical basis. We used, as a first approximation, a first-order low-pass filter as a movement-detector filter in both movement-detector subunits. According to experimental measurements, ${ }^{51,52} 100$ msec was chosen for the filter time constant. The spatial wavelength was chosen to be 36 times the sampling base, which is similar to that used in the experiments. Since there is good experimental evidence that the background luminance is removed to a high degree (but not entirely!) at processing stages peripheral to movement detection, ${ }^{44}$ the effective contrast $\Delta I / I$ at the movement-detector input was set in the simulations to as high as 2.8. The relative contributions of the different frequency components then were adjusted to the values obtained in the experiments by varying the gain of the detector subunit that is subtracted. Otherwise, the stimulus conditions were chosen as in the corresponding experiments.

The movement-detector responses to motion in the preferred direction, to motion in the null direction, and to field flicker stimulation are displayed in Fig. 4. Although there are differences in some details, the time courses of the model simulations are quite similar to their experimental counterparts. The main differences between computer simulations and experimental results relate to the phase relationship between the stimulus and the corresponding response. This difference is most pronounced for field flicker stimulation. It should be noted, however, that we did not intend to fit the experimental data as closely as possible. This certainly could have been done by assuming filters of higher order or by using combinations of different filters in both branches of each movement-detector subunit. Instead, we tried to ac- count for the most-prominent features of the time course of the HS-cell response with as few assumptions as possible. As has been shown here, this is in fact possible.

\section{CONSEQUENCES FOR THE INTEGRATED MOVEMENT-DETECTOR RESPONSE}

As one of the main results of Section 3, it was concluded that the outputs of the two subunits of the movement detectors in the fly's visual system are not perfectly balanced. This result has several consequences with respect to the specific information about the visual surround that is represented by the movement-detection system. These consequences pertain to (1) the direction selectivity of the movement-detection system and (2) its responsiveness to stationary stimuli with temporally modulated brightness. Both consequences are analyzed in what follows. They can be seen most easily in the spatially integrated movement-detector responses. In our experiments we therefore exploit the fortunate situation that the HS cell integrates the outputs of large retinotopic arrays of movement detectors and thus allows us to study in one and the same cell the responses of individual movement detectors (by the slit technique) as well as the spatially integrated response.

\section{A. Directional Selectivity is Not Constant}

The predicted responses of an array of movement detectors to motion in the preferred direction $\left(\overline{R^{\mathrm{p}}}\right)$ and the null direction $\left(\overline{R^{\mathrm{n}}}\right)$ are obtained by spatially integrating the equations describing the single-detector responses [Eqs. (6)-(13)] with respect to the spatial variable $\varphi$ : 


$$
\begin{aligned}
\overline{R^{\mathrm{p}}}(t)= & I^{2}(1-g)+(1 / 2) \Delta I^{2}\left(\operatorname { c o s } ( 2 \pi \Delta \varphi / \lambda ) \left\{F_{1}(\omega) \cos \left[\phi_{1}(\omega)\right]\right.\right. \\
& \left.-g F_{2}(\omega) \cos \left[\phi_{2}(\omega)\right]\right\}-\sin (2 \pi \Delta \varphi / \lambda) \\
& \left.\times\left\{F_{1}(\omega) \sin \left[\phi_{1}(\omega)\right]+g F_{2}(\omega) \sin \left[\phi_{2}(\omega)\right]\right\}\right)
\end{aligned}
$$

and

$$
\begin{aligned}
\overline{R^{\mathrm{n}}}(t)= & I^{2}(1-g)+(1 / 2) \Delta I^{2}\left(\operatorname { c o s } ( 2 \pi \Delta \varphi / \lambda ) \left\{F_{1}(\omega) \cos \left[\phi_{1}(\omega)\right]\right.\right. \\
& \left.-g F_{2}(\omega) \cos \left[\phi_{2}(\omega)\right]\right\}+\sin (2 \pi \Delta \varphi / \lambda) \\
& \left.\times\left\{F_{1}(\omega) \sin \left[\phi_{1}(\omega)\right]+g F_{2}(\omega) \sin \left[\phi_{2}(\omega)\right]\right\}\right) . \quad(26 \mathrm{~b})
\end{aligned}
$$

Now, the responses are no longer time dependent. It is obvious that $R^{\mathrm{p}}$ and $R^{\mathrm{n}}$ each consist of two components. One component is common to both the response in the preferred direction and that in the null direction and is thus independent of the direction of motion:

$$
\begin{aligned}
\overline{R_{\mathrm{di}}}= & (1 / 2)\left(\overline{R^{\mathrm{p}}}+\overline{R^{\mathrm{n}}}\right)=I^{2}(1-g)+(1 / 2) \Delta I^{2} \cos (2 \pi \Delta \varphi / \lambda) \\
& \times\left\{F_{1}(\omega) \cos \left[\phi_{1}(\omega)\right]-g F_{2}(\omega) \cos \left[\phi_{2}(\omega)\right]\right\} .
\end{aligned}
$$

The other component has the same amplitude but the opposite sign for motion in opposite directions:

$$
\begin{aligned}
\overline{R_{\mathrm{ds}}}= & (1 / 2)\left(\overline{R^{\mathrm{p}}}-\overline{R^{\mathrm{n}}}\right)=-(1 / 2) \Delta I^{2} \sin (2 \pi \Delta \varphi / \lambda) \\
& \times\left\{F_{1}(\omega) \sin \left[\phi_{1}(\omega)\right]+g F_{2}(\omega) \sin \left[\phi_{2}(\omega)\right]\right\} .
\end{aligned}
$$

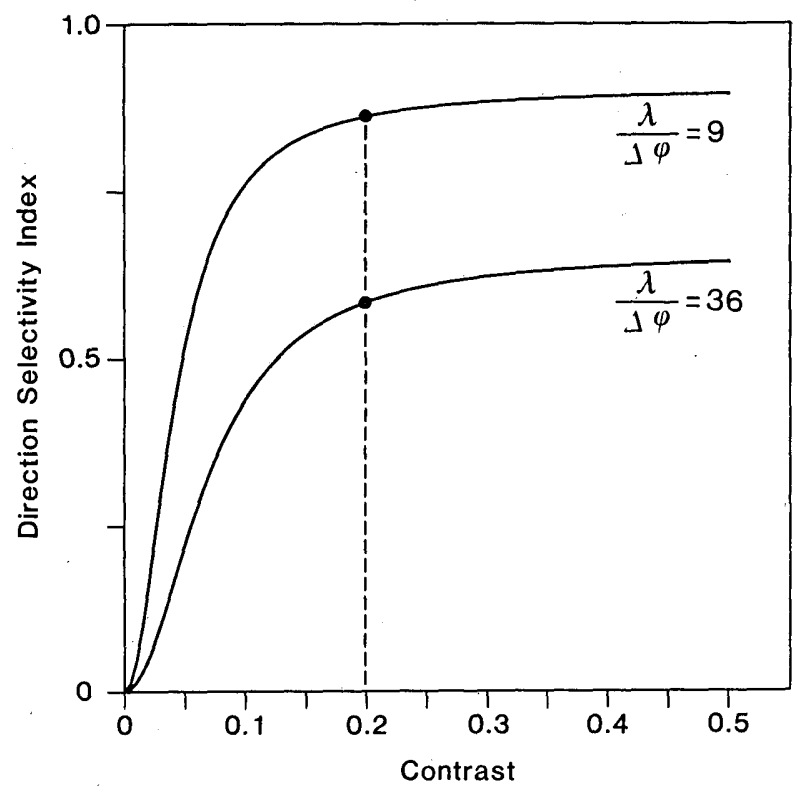

Fig. 5. Computer simulation of the contrast dependence of the direction-selectivity index $I_{\mathrm{ds}}$ of a correlation-type movement detector that is unbalanced at its subtraction stage. The parameter in these simulations is the spatial wavelength $\lambda$ of the stimulus pattern, which is given in units of the angular distance $\Delta \varphi$ between the two movement-detector input channels. $I_{\mathrm{ds}}$ was calculated according to Eq. (30). All model parameters were chosen as in the computer simulations shown in Fig. 4. Again, the background luminance was subtracted to a high degree from the input signals, so that a pattern contrast of $100 \%$ corresponds to an effective contrast of 14 at the movement-detector input. For both spatial wavelengths, $I_{\mathrm{ds}}$ steeply increases at low contrasts. For pattern contrasts greater than $20 \%$ the curves approach a constant level. $I_{\mathrm{ds}}$ is smaller for the larger spatial wavelength than for the smaller one.

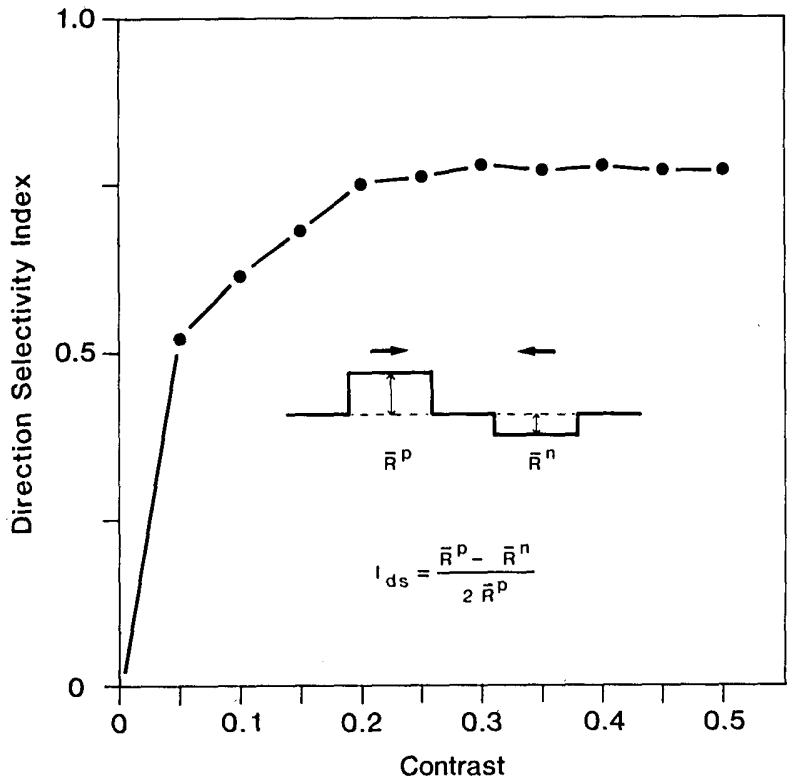

Fig. 6. Contrast dependence of the direction-selectivity index of the HS cell. The animal was stimulated with a vertical sine-wave grating of variable contrast. The pattern was moved alternately for 4 sec from the front to the back, i.e., in the cell's preferred direction, and from the back to the front, i.e., in the cell's null direction. The temporal frequency was $1 \mathrm{~Hz}$; the spatial wavelength was $17^{\circ}$. The mean responses to motion in the preferred direction $\left(R^{p}\right)$ and in the null direction $\left(R^{\mathrm{n}}\right)$ were determined by calculating the mean deviation of the membrane potential from its resting level during the second half of the stimulation time. The average was taken only during this time so that the results would not be affected by potential response transients (although these were not pronounced under these stimulus conditions). The direction-selectivity index $I_{\mathrm{ds}}$ was calculated as is indicated by the formula. The data are averages from four flies and a total of 36 stimulus presentations. For all data points, the standard errors of the mean are smaller than 0.04. For contrasts as much as 0.20 , the direction-selectivity index increases with pattern contrast and then reaches its final plateau level.

With $g=1, F_{1}(\omega)=F_{2}(\omega)=F(\omega)$, and $\phi_{1}(\omega)=\phi_{2}(\omega)=\phi(\omega)$, i.e., with exactly balanced detector subunits, the directioninsensitive component disappears, leading to an antisymmetrical response:

$$
\overline{R^{\mathrm{p}}}=-\overline{R^{\mathrm{n}}}=-\Delta I^{2} F(\omega) \sin [\phi(\omega)] \sin (2 \pi \Delta \varphi / \lambda) .
$$

The term $\sin (2 \pi \Delta \varphi / \lambda)$, describing the dependence of the detector response on the ratio between the sampling base $\Delta \varphi$ and the spatial wavelength of the pattern $\lambda$, is known as the geometrical interference term. ${ }^{23,24}$ It can be derived from Eqs. (27) and (28) that the direction-independent and direction-dependent response components are determined in different ways by the different stimulus parameters such as pattern contrast and spatial wavelength. This means also that the property of the movement-detection system to respond to motion in a direction-selective manner is not in variant with respect to changes in the stimulus conditions. It is obvious that direction selectivity is related to the difference of the responses to motion in the preferred and null directions. We therefore introduce a direction-selectivity index $\left(I_{\mathrm{ds}}\right)$ :

$$
I_{\mathrm{ds}}=\frac{\overline{R^{\mathrm{p}}}-\overline{R^{\mathrm{n}}}}{2 \overline{R^{\mathrm{p}}}}
$$



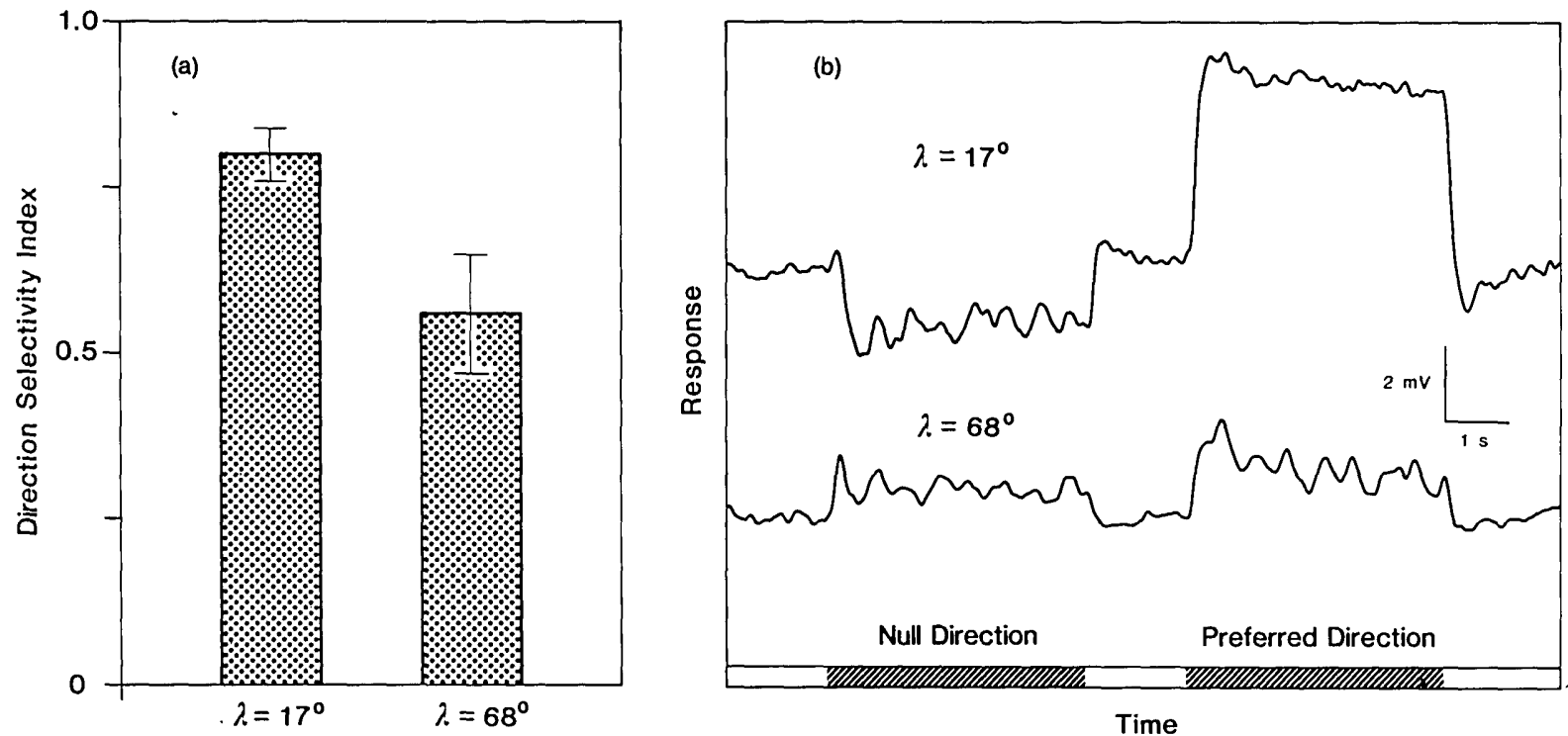

Fig. 7. Dependence of the direction selectivity of the HS cell on the spatial wavelength of the stimulus pattern. The stimulus pattern was a sine-wave grating with a contrast of 0.20 that moved with a temporal frequency of $1 \mathrm{~Hz}$ either in the cell's preferred direction or in its null direction. (a) The mean direction-selectivity index is shown for a spatial wavelengths of $17^{\circ}$ and $68^{\circ}$. The direction-selectivity index was calculated as described for Fig. 6 . The data were obtained from eight flies and a total of 65 presentations of the stimulus program. The bars indicate the standard error of the mean. (b) An extreme example in which the directionalty of the HS cell changes most dramatically. The intracellularly recorded responses are plotted as a function of time and are averages from a total of 15 stimulus presentations each. The patterns had a wavelength of either $68^{\circ}$ or $17^{\circ}$. The direction and time of stimulus motion is indicated at the bottom of the diagram. The direction selectivity is smaller for patterns with a large spatial wavelength than for those with a small wavelength. The response to motion in the null direction may even reverse its sign when the spatial wavelength is increased, leading to almost the same amplitude as that of the response to motion in the preferred direction and consequently to only a small direction selectivity.

which varies between 0 and 1 . These extreme values correspond either to $\overline{R^{\mathrm{p}}}=\overline{R^{\mathrm{n}}}$ (i.e., the system is not direction selective at all) or to $\overline{R^{p}}=-\overline{R^{n}}$ (i.e., the system responds with the same amplitude but with opposite sign).

Two predictions concerning the stimulus dependence of $I_{\mathrm{ds}}$ can be derived from Eqs. (30) and (26). (1) The direction selectivity is expected to increase with increasing pattern contrast. (2) The direction selectivity should decrease when the spatial wavelength of the stimulus pattern increases. These qualitative predictions are illustrated in Fig. 5. The direction-selectivity index was calculated for two different spatial frequencies as a function of pattern contrast by using the same parameters as in the computer simulation shown in Fig. 4. Of course, the direction selectivity depends on the pattern wavelength and contrast only as long as the subunits of the movement detector are not perfectly balanced. Otherwise, one always gets $I_{\mathrm{ds}}=1$.

To test whether the direction selectivity of the HS cell depends on pattern contrast, the responses to motion from the front to the back and from the back to the front were measured at different contrasts ranging from $\Delta I / I=0.05$ to $\Delta I / I=0.50$. The mean luminance was held constant at $\sim 25$ $\mathrm{cd} / \mathrm{m}^{2}$. From the data the direction-selectivity index was calculated according to Eq. (30) and plotted in Fig. 6 as a function of contrast. The direction-selectivity index increases steeply for pattern contrasts smaller than 0.20 . For higher contrasts the index does not increase further and approaches a constant level. The qualitative features of the dependence of tho diroction-soloctivity index on pattern contrast are in excellent agreement with the theoretical predictions (compare Figs. 5 and 6 ).

To test the prediction that the direction selectivity of the
HS cell depends on the spatial wavelength of the pattern, the responses to motion in the preferred and null directions were measured at a contrast of 0.20 for two different spatial wavelengths. As is shown in Fig. 7(a), the mean direction-selectivity index is considerably higher for $\lambda=17^{\circ}$ than for $\lambda=$ $68^{\circ}$. (This is just what has been predicted, as is illustrated by the filled circles at contrast of 0.20 in Fig. 5.) An extreme example is shown in Fig. 7(b). Here two features are particularly striking: (1) The response to motion in the preferred direction is much smaller when the fly is stimulated with the $68^{\circ}$ grating. This is expected because of the dependence of the direction-selective component of the response on the spatial wavelength [see Eq. (28)]. (2) The response to motion in the null direction reverses its sign after increasing the spatial wavelength of the stimulus pattern. Since, in the example shown in Fig. 7(b), the responses to motion in the preferred and null directions have the same sign and almost the same amplitude, the cell has nearly lost its direction selectivity. From these results it can be concluded that the directionality of a movement-sensitive system depends on the properties of the stimulus pattern, just as has been predicted by the movement-detector theory.

\section{B. Sensitivity to Stationary Time-Modulated Patterns} It has been concluded in Section 3 that a movement detector of the correlation type is sensitive to stationary patterns whose brightness is temporally modulated, unless its two subunits are balanced precisely. With the slit paradigm this flicker sensitivity could already be established. It can be analyzed further when, instead of the single-movement-detector response, the spatially integrated output of an array of detectors is examined. Two different flicker stimuli will 
be considered here, since they are particularly illustrative with respect to the motion-detection mechanism: (1) The brightness of a stationary sine-wave grating was modulated sinusoidally in time; this stimulus condition is referred to as counterphase flicker. (2) The brightness of a spatially homogeneous field was modulated sinusoidally in time. As in the slit paradigm, this stimulus is called field flicker.

In the counterphase flicker situation (as indicated by the superscript $\mathrm{cf}$ ), the right and left input signals of a movement detector are given by

$$
A_{1}{ }^{\mathrm{cf}}(t)=I+\Delta I \sin (\varphi) \sin (\omega t)
$$

and

$$
A_{2}{ }^{\mathrm{cf}}(t)=I+\Delta I \sin (\varphi-2 \pi \Delta \varphi / \lambda) \sin (\omega t) .
$$

After calculating the signals by a method analogous to that used for the responses to motion (see Subsection 3.A) and then spatially integrating over an array of movement detectors covering an integral number of spatial wavelengths, one

obtains the response of the movement-detection system to counterphase flicker stimulation,

$$
\begin{aligned}
\overline{R^{\mathrm{cf}}}(t)= & I^{2}(1-g)+(1 / 4) \Delta I^{2} \cos (2 \pi \Delta \varphi / \lambda) \\
& \times\left\{F_{1}(\omega) \cos \left[\phi_{1}(\omega)\right]-g F_{2}(\omega) \cos \left[\phi_{2}(\omega)\right]\right. \\
& \left.+g F_{2}(\omega) \cos \left[2 \omega t+\phi_{2}(\omega)\right]-F_{1}(\omega) \cos \left[2 \omega t+\phi_{1}(\omega)\right]\right\} .
\end{aligned}
$$

The response consists of two components, one being independent of time and the other varying at twice the temporal frequency of the stimulus pattern. Hence all the power of the time-dependent part of the response is contained in the second harmonic rather than in the fundamental frequency.

In contrast to counterphase flicker stimulation, all photoreceptors receive the same input signals in the field flicker situation (see also Subsection 3.A, Eq. (14)]. The spatially integrated response of an array of movement detectors to field flicker stimulation is then the same, apart from a constant factor, as the response of a single detector. Both can

\section{Field Flicker}
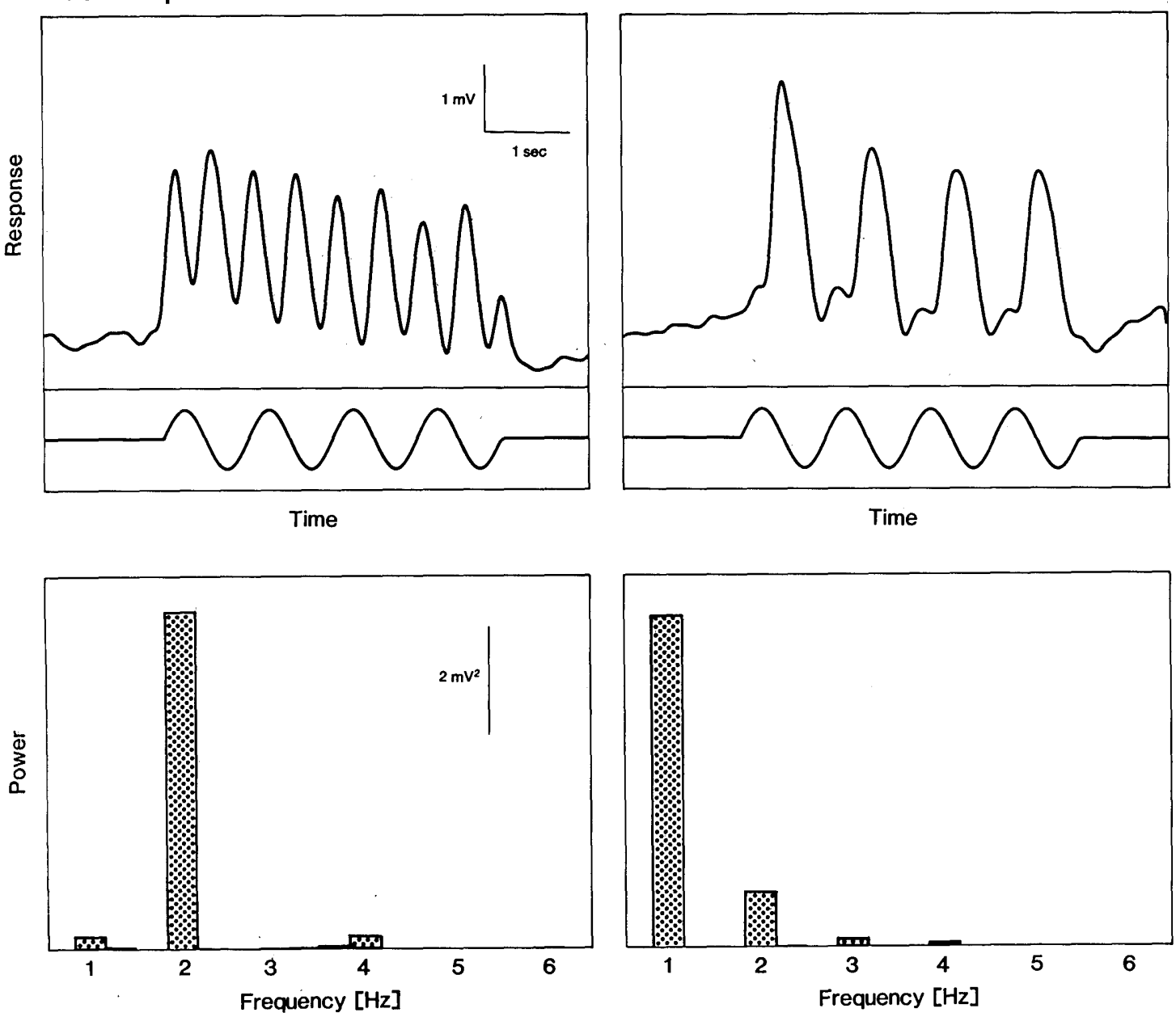

Fig. 8. Responses of the HS cell to (left) counterphase and (right) field flicker stimulation. In the case of counterphase flicker stimulation, the stimulus pattern was a sine-wave grating with a spatial wavelength of $17^{\circ}$. In the case of field flicker stimulation, the monitor was spatially uniform. The temporal frequency was $1 \mathrm{~Hz}$, and the contrast was 0.20 . The upper diagrams are the time-dependent responses as averaged from 20 presentations of the stimulation program. The brightness modulation in the middle of the screen is indicated below the timedependent plots. The bottom diagrams are the power spectra of the time-dependent response profiles. As in Fig. 2 only the three last response cycles were used for analysis. During counterphase flicker stimulation the response is modulated essentially with the second harmonic of the temporal frequency of the stimulus, whereas during field flicker stimulation the fundamental frequency predominates. 
thus be described by the same formal expression [see Eqs. (17)-(24)]. The response is modulated in time with the temporal frequency of the stimulus, and there is also a second-harmonic component. The qualitative differences between the responses to counterphase and field flicker stimulation allow us to test the movement-detector model further.

Figure 8 shows the result of an experiment in which the HS cell was stimulated alternately by counterphase flicker and by field flicker. The different time courses of the responses under these different stimulus conditions are immediately striking. Whereas the response to counterphase flicker is modulated essentially with twice the temporal frequency of the stimulus pattern, the response to field flicker stimulation follows mainly the temporal frequency. These conclusions are supported by the power spectra of the responses, as shown below the time-dependent curves. For counterphase flicker almost all the power is contained in the second harmonic, whereas for field flicker stimulation there is, apart from a small second-harmonic component, mainly a large component at the fundamental frequency. These experimental results are thus in close agreement with the model predictions. The characteristic time courses of the responses support in a specific way the existence of a multiplicationlike interaction in the movement-detection system of the fly.

\section{DISCUSSION}

\section{A. Mechanism of Motion Detection in the Visual System of the Fly}

Motion detectors of the correlation type were used successfully to explain various aspects of motion vision in species as different as insects ${ }^{2,8,9,20-25,44,45,48,49}$ and humans. ${ }^{13-18}$ The core of this motion-detection scheme, as well as of its mathematical equivalents, ${ }^{17,19}$ is formed by a quadratic nonlinearity. In the case of a correlation-type movement detector, the signals originating from two spatially displaced input channels are multiplied. This is done after they have been delayed with respect to each other. To enhance its direction selectivity, a movement detector of the correlation type consists of two mirror-symmetrical subunits whose outputs are finally subtracted. In its mathematically ideal form, the movement detector has two principal characteristics: (1) It is antisymmetrical; i.e., it responds with the same amplitude but opposite signs to motion in its preferred and null directions. (2) It responds only to motion and is completely insensitive to stationary patterns, even if these are modulated in time. Do biological movement detectors conform to this mathematically perfect scheme? If there are deviations, how can the realizations of movement detectors in a nervous system be characterized, and to what extent do they approximate the mathematically perfect scheme?

These questions were analyzed here for the motion detection system of the fly. So far, most evidence in favor of a correlation-type movement detector has been based on the analysis of spatially and/or temporally integrated movement-detector responses (see Section 1). However, it is obvious that more specific information can be obtained by recording the time-dependent responses of individual movement detectors. This has been done here by using a simple technique. We recorded from a direction-selective motionsensitive interneuron in the fly's brain, which spatially integrates a large number of retinotopically organized local- movement detectors. The method used to derive the response properties of individual movement detectors from this type of neuron was to stimulate a large number of movement detectors virtually in synchrony. This was done by exposing the eye, through a small vertical slit, to an appropriate stimulus pattern. So far, only the steady-state responses of local-movement detectors were taken into account. However, the slit technique can also be applied to investigate whether even the transient properties of individual movement detectors can be accounted for by the movement-detector theory as outlined here. For the transient responses of a spatially integrated array of detectors this was shown previously: 44,45

The slit technique was applied recently in a behavioral study in which the visually induced optomotor yaw torque response was used as an indicator of the performance of the fly's motion-detection system. ${ }^{46}$ In contrast to a neuronal analysis, in behavioral experiments it is relatively difficult to define the zero-response level of the movement-detection system. For this reason, instead of only one slit, two slits symmetrically arranged in front of the two eyes were used, and the stimulus pattern was moved alternately clockwise and counterclockwise. Under these conditions, the zero response level could be determined easily as a line of symmetry between the responses to the oppositely directed motions. In this way, however, any direction-independent component of the movement-detector responses inevitably remained unnoticed. These components, however, proved to be essential in characterizing the exact formal nature of the computations performed by the detector.

Here we have demonstrated two characteristic features of the movement detectors in the fly's visual system: (1) They respond with different time courses and amplitudes to motion in their preferred and null directions and thus are not perfectly antisymmetrical. This finding was interpreted as an indication that the two movement-detector subunits are not balanced. Instead, the one that contributes with a positive sign to the overall response of the detector has been concluded to have the larger gain. Formally, this imbalance can be attributed either to an imbalance in the detector's subtraction stage or to slightly different properties of the temporal filters in its input channels. (2) The multiplication that represents the essential interaction between the two movement-detector input channels appears to be realized almost perfectly in the fly's brain. This conclusion holds for contrasts at least as high as $30 \%$. For higher contrasts it is difficult to draw definite conclusions in this respect, since other nonlinearities, such as saturation phenomena in the input channels to the movement detector, ${ }^{44}$ may affect the response. For instance, these may lead to specific motion illusions, as was reported by Bülthoff and Götz for both humans and flies. ${ }^{53}$ The finding that the essential nonlinearity of the movement detector is a multiplication is consistent with other electrophysiological evidence on the fly's visual system. In studies on small-field motion-sensitive visual interneurons in the second visual ganglion, using moving sine-wave gratings, ${ }^{54}$ and in studies in another large-field visual interneuron in the third visual ganglion, using white-noise techniques, ${ }^{55,56}$ it was concluded that nonlinearities above second order do not contribute significantly to the response. It should be noted that the socalled gradient model, ${ }^{3-7}$ another type of motion-detection scheme (see Section 1), cannot explain the experimental 
findings of the present study. Most notably, the temporal modulations of the local-detector responses to a moving stimulus pattern are not predicted by this model, at least in its pure mathematical form.

\section{B. Alternative Approaches to Study the Properties of Individual Movement Detectors}

A movement detector can be stimulated either by real motion, i.e., drifting stimulus patterns, or by apparent motion, i.e., local stimuli projected separately in a sequential order on its input channels. It is often claimed that the latter method allows one to probe directly the computations performed by the movement detector. In this way, the latter method is assumed to monitor the activity of individual movement detectors regardless of whether one records from a spatially integrating unit. This approach was pioneered by Barlow and Levick ${ }^{57}$ in the rabbit retina and was applied subsequently in various studies on motion-sensitive neurons at various levels of the vertebrate visual system ${ }^{58-63}$ but also in insects. ${ }^{12,55,56,64-66}$ Insects lend themselves especially well to this kind of analysis since, because of the ommatidial structure of the insect eye, it is possible to stimulate even single photoreceptors individually.

This approach may be quite useful, if one is interested essentially in studying the spatial and temporal factors underlying the motion-detection process by varying the spatial or temporal separation between the two stimuli. Despite its appeal for many investigators, this approach may have some disadvantages. If one is interested in how the nonlinear interaction of the movement-detector input channels can be characterized formally, the responses obtained in this way are difficult to interpret from an analytical point of view. This is mainly because stimuli that lead to pronounced temporal discontinuities at the input site of the visual system are much more liable to have distortions of their time course because of signal processing peripheral to the movement detector than are stimuli, such as sine waves, that are modulated only smoothly. Distortions in the time domain, for instance, are almost inevitable in all biological vision systems, since the photoreceptors act as a kind of low-pass filter, thus considerably smoothing flashed stimuli. Moreover, the retinal image may be initially spatially bandpass filtered before being passed to the movement detectors. This is achieved, for instance, in the vertebrate retina, by ganglion cells that have an excitatory center and an inhibitory surround. When the movement detector is now stimulated by small localized apparent-motion stimuli, the resulting responses will depend on whether the stimuli fall on the center or on the surround of the movement-detector input elements. ${ }^{59,67,68}$ Hence the qualitative features of the movement-detector output are determined essentially by the preprocessing of the movement-detector input signals. As a consequence, it is difficult to separate these effects from the consequences of the nonlinear interaction between the two movement-detector input channels. In contrast, if sinewave gratings are used for the analysis and the spatial preprocessing is approximately linear (as is usually assumed, at least for the retinal X ganglion cells; see e.g. Refs. 69 and 70), the movement-detector input signals are again sine-wave functions. On this basis we can derive theoretical predictions even analytically that can then be challenged experimentally. The problem of altering the shape of the movement-detector input signals may be even greater when there are nonlinearities, such as saturation phenomena, in the input channels. These may severely affect the time course of the motion-detector responses. This problem appears to be a particular problem in the insect visual system, in which single-receptor stimulation has usually been done with darkadapted animals and high-contrast flashes in order to obtain sufficiently large responses. ${ }^{12,64-66}$ In this respect, our slit paradigm is advantageous, since here one can stimulate a large number of detectors virtually in synchrony, which allows one to use less extreme stimulus conditions.

\section{Consequences of an Unbalanced Movement Detector} From our experiments it has been concluded that the two subunits of the movement detector are not perfectly balanced. As a consequence, the movement detector no longer is strictly selective for motion but also responds, at least to some extent, to brightness modulations of stationary patterns. Similar responses to stimulation with counterphase flicker as described here were obtained in another motionsensitive cell in the third visual ganglion ${ }^{71}$; field flicker stimulation, however, was not tested in this study.

It is interesting to note that in the fly a specific sensitivity to flicker stimulation has been known for a long time at the behavioral level. ${ }^{72-76}$ This finding has been interpreted as evidence in favor of an additional channel, in parallel to the movement-detection system, that indicates to the visual system the position of an object in the visual field. ${ }^{30,77}$ In contrast, in the present study it has been demonstrated that the movement detectors as they are used in the fly's brain inevitably are to some extent sensitive to flicker stimuli. However, the experimental evidence is, so far, too inconclusive to permit a decision whether the behavioral responses to flicker stimulation are just the by-product of the motiondetection system or whether there is a separate system involved in their control.

The amount of selectivity of the movement-detection system for the direction of pattern motion has been shown here to depend on the spatial wavelength of the stimulus pattern. As can be derived from Eq. (30), the direction selectivity has its optimum for spatial wavelengths of four times the distance between the movement-detector input channels and decreases with increasing pattern wavelengths. This suggests that for natural stimuli the direction selectivity of the motion-detection system might be sufficiently high, as long as it has sufficient energy in the high-spatial-frequency range. Hence it appears that under most normal conditions the imperfection in the subtraction stage of the movement detector does not have deleterious effects on the performance of the system.

\section{Similar Findings in Other Species}

Results of psychophysical experiments on humans, ${ }^{14-16,18,78}$ as well as electrophysiological data on the vertebrate visual cortex, ${ }^{60,63}$ suggest that movement detectors based on a quadratic nonlinearity, such as correlation-type movement detectors, may play a decisive role in motion vision throughout the animal kingdom. This implies, however, that motionsensitive interneurons in the vertebrate visual system should show response characteristics similar to those found for the fly in the present study.

Most studies on motion-sensitive neurons in which sinewave gratings are used as stimulus patterns seem to be concerned mainly about whether a particular cell summates 
over its retinotopic input in a linear or nonlinear way. They do not focus on the question of how the responses are determined by the nonlinearities of the motion-detection mechanism. ${ }^{79-83}$ As far as we know, it has never been discussed in the vertebrate literature, for instance, that any motion-sensitive cell inevitably has a spatial-frequency dependence with a clear optimum without assuming spatial bandpass filters in the movement-detector input channels. The wavelength dependence is determined by the geometry of the detector input channels and the detection mechanism. For a mathematically perfect correlation-type of movement detector the wavelength dependence is independent of the temporal frequency of stimulus motion and can be described by the so-called geometrical interference term $\sin (2 \pi \Delta \varphi / \lambda)$ [see Eqs. (28) and (29)], in which $\lambda$ and $\Delta \varphi$ denote the spatial wavelength of the stimulus pattern and the angular separation of the two movement-detector input channels, respectively. ${ }^{23,24}$ One must take this term into account when interpreting the wavelength dependence of motion-sensitive cells even if not all spatial frequencies are transmitted equally well to the movement-detector input, either because of the contrast transfer properties of the eye ${ }^{84}$ or by some sort of neuronal bandpass filtering. ${ }^{16}$

Nevertheless, there are studies on responses to moving sine-wave gratings whose results can be compared with the predictions of the movement-detector theory. It seems to be accepted generally that direction-selective motion-sensitive cells in the visual cortex can be classified roughly into two categories. ${ }^{85,86}$ In one category are cells in which the responses are modulated while a grating passes through their receptive field. The main component of the modulation frequency seems to correspond to the temporal frequency of the stimulus. In the other category are neurons that do not show these response modulations. This dichotomy of motion-sensitive cells is usually related to the simple-complex classification scheme of cortical neurons, as is based essentially on the spatial layout of the cells' receptive fields. As has been shown here, an individual movement detector is expected to show temporally modulated responses. However, these response modulations disappear if the signals of an array of movement detectors covering at least one spatial period of the stimulus pattern are spatially integrated. If this integration field is matched to the optimal spatial wavelength of the cell, the resulting receptive field of an integrating unit can nevertheless be rather small. Whereas simpletype cortex cells are assumed to have spatially separate on and off regions, complex-type cells appear to be different in this respect, since they have homogeneous on-off receptive fields. ${ }^{80,81,87,88}$ Let us further assume that the input channels to the nonlinear interaction that endows the cell with its motion sensitivity have bandpass filter characteristics and hence receptive fields with separate spatial on and off regions. The available evidence then supports the conclusion that the motion-sensitive cells that show a modulated response pattern do not integrate much along their preferred direction over motion-sensitive input elements. In contrast, the cells that do not reveal modulated responses can be interpreted to integrate over such elements that are spatially displaced along the cell's preferred direction. To our knowledge, there are no published data that would critically challenge this interpretation. One critical test, for instance, would be, as has been done here for the fly's motion-detec- tion system, to compare the temporal-frequency components in the responses with motion in the cell's preferred and null directions.

Owing to the conclusions drawn in the present paper, substantial evidence in favor of this interpretation can, at least in principle, be obtained by using flicker stimulation. In the cat suprasylvian cortex, for instance, where most of the cells are directionally selective for motion, flicker sensitivities are found that agree quite well with the predictions of the present paper. ${ }^{86}$ Cells that show an unmodulated response to motion respond to counterphase flicker essentially with a second-harmonic modulation irrespective of the spatial phase of the stimulus pattern. This is what is expected from a cell that integrates spatially over a sufficiently large array of movement detectors. In contrast, neurons that respond with a modulated activity to moving gratings have a strong first-harmonic modulation in their response to counterphase flicker stimuli. The relative contributions of the first and second harmonics, however, depend on the spatial phase of the stimulus pattern. Although counterphase flicker stimuli were not used in our slit paradigm, it is obvious that this result is predicted by the movement-detector theory, because the stimulus conditions for an individual movement detector depend, during counterphase flickering, on the spatial phase of the pattern. In one extreme phase both input channels of the detector receive approximately the same time-dependent input signals. This corresponds to our field flicker situation and should lead to responses with a strong first-harmonic component. In the other extreme phase, the two input channels are stimulated in counterphase. On the basis of movement-detector theory, this should lead to a response modulation with twice the temporal frequency of the stimulus. These findings ${ }^{86}$ are consistent with the data obtained in other studies on cortical neurons. ${ }^{80,81,83}$ In the latter studies, however, it usually was not specified whether the tested cells were motion sensitive. Those results therefore should be interpreted only with caution in the present context.

Despite all these qualifications, the responses of motionsensitive cells in the mammalian visual cortex to grating stimuli agree quite well with our findings on the motiondetection system of the fly. This is true not only for motion stimuli but also for stimuli in which only the brightness of a stationary pattern is modulated in time.

\section{E. Cellular Implementation of Movement Detectors}

The movement-detector model as shown in Fig. 1 is an algorithmic model that specifies the principal computations performed by the detector. It does not specify the actual neuronal implementation of the movement detector in the fly's brain. Various implementation schemes might be possible.

A cellular implementation of the subtraction stage of the movement-detector model is, in principle, quite simple. One needs only to assume that the outputs of the two detector input channels control an excitatory synapse and an inhibitory synapse. In this case, however, the subtraction leads to a perfectly antisymmetrical detector response only if the output signals of the two detector subunits control the same conductance changes and have the same, although oppositely directed, driving forces, i.e., have equilibrium potentials differing from the resting potential of the cell by the same amount. Only then are the two detector subunits 
Preferred Direction

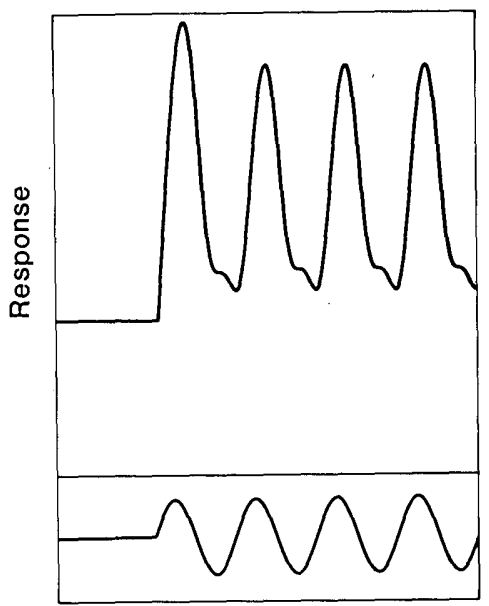

Null Direction

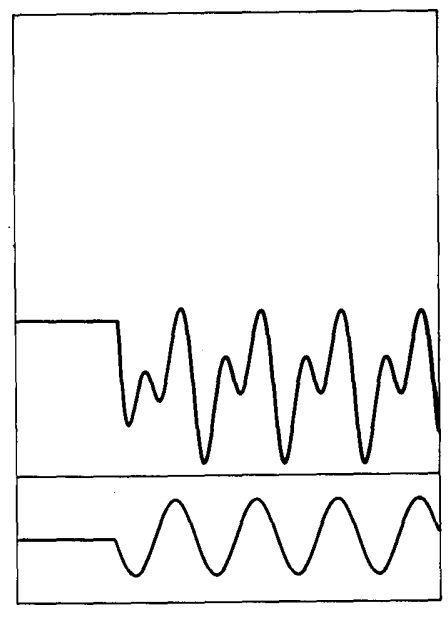

Field Flicker

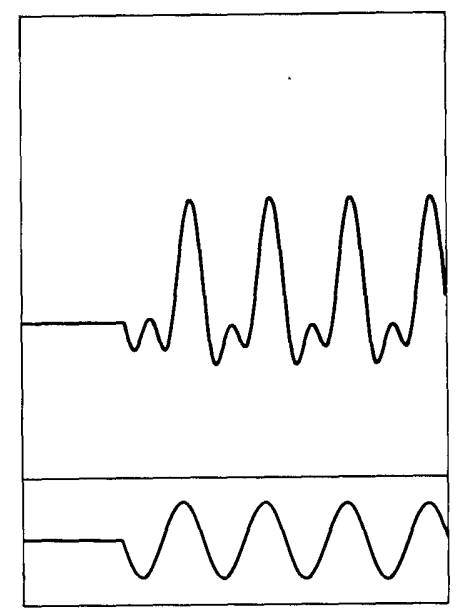

Time

Fig. 9. Computer simulation of the responses of a movement detector of the correlation type to motion in its preferred and null directions as well as to flicker stimulation. All model and stimulus parameters are as in the simulation shown in Fig. 4, except that the subtraction stage was implemented in cellular terms. It was assumed that the two subunit outputs control different ionic channels with equilibrium potentials that are positive $\left(E_{e}\right)$ and negative $\left(E_{i}\right)$ with respect to the resting potential and with conductances $g_{e}$ and $g_{i}$, respectively. $g_{e}$ and $g_{i}$ each are assumed to consist of a time-independent component and a time-dependent component that represents the output signal of the respective detector subunit. In addition, a constant leak conductance $g_{1}$ with equilibrium potential $E_{1}$ was assumed. The time-dependent output of a movement detector was then calculated according to the standard formula $V(t)=\left(g_{e} E_{e}+g_{i} E_{i}+g_{1} E_{1}\right) /\left(g_{e}+g_{i}+g_{1}\right)$. For the different parameters the following numerical values were chosen: $E_{e}=-10, E_{i}=-70, E_{1}=-50, g_{1}=20$; the sum of the time-independent components of $g_{e}$ and $g_{i}$ was 40 . In this way the two detector subunits became unbalanced and sensitive to flicker stimulation. The computed responses are virtually indistinguishable from those shown in Fig. 4. The type of physiological subtraction used in this model simulation thus appears to be sufficient to account for the experimentally determined asysmmetries in the organization of the fly's movement-detection system.

perfectly balanced, and only then do they not respond, e.g., to flicker stimuli. Although these assumptions cannot be tested in the fly's visual system, it is obviously unlikely that they would be realized perfectly in any biological system. Moreover, our findings clearly indicate that the two detector subunits are, in fact, unbalanced. A natural way to account for this is the common assumption that the driving force of the inhibitory channel is smaller than that of the excitatory one. In this way, the excitatory channel is endowed with a higher weight. If the visually induced membrane potential changes do not deviate too much from the cell's resting potential, this weighting factor is approximately constant, as has been derived from our experiments on the HS cells of the fly. A computer simulation based on this type of cellular subtraction is shown in Fig. 9. All other assumptions concerning the different parameters characterizing the movement detector are the same as in the model simulation shown in Fig. 4. The time courses of the responses to motion in the preferred and null directions, as well as those of the responses to flicker stimulation, are virtually identical to those shown in Fig. 4, in which, instead of a physiological subtraction, a mathematical one was used. This similarity demonstrates that physiological subtraction is sufficient to account for our experimental data and therefore is a plausible reason for the movement detectors in the fly's visual system to be unbalanced. Since the physiological reasons for this type of imbalance cannot be avoided, it is difficult for any biological movement-detection system to escape from being sensitive, at least to some degree, to flicker stimulation. Nevertheless, as has been shown above, this does not substantially deteriorate the performance of this type of motion-detection system in extracting motion information under most naturally occurring conditions.
With respect to the nonlinear interaction of the two detector input channels, different proposals have been made that are plausible in neuronal terms. ${ }^{10,26,89,90}$ The so-called shunting inhibition model assumes that the two movementdetector input channels synapse on a common element, one with an excitatory synapse and the other with an inhibitory synapse. This inhibition is mediated by a synapse with a reversal potential close to the resting potential of the cell. ${ }^{10,89}$ The so-called threshold model also assumes that the movement-detector input channels synapse on a common element. In this model this combination may be linear. The essential nonlinearity is assumed to be due to a threshold operation. ${ }^{26}$ To what extent do these different types of nonlinear interaction approximate a multiplication? Owing to our present findings, this is a precondition for these cellular models to be plausible candidates for motion detection in the fly. Grzywacz and $\mathrm{Koch}^{26}$ analyzed this question by model simulations and showed that both cellular models approximate a quadratic interaction of the two detector input channels only poorly. This conclusion, however, should be drawn only with some caution, since these computer simulations ${ }^{26}$ were done under special assumptions, concerning the phase difference of the signals in the two input channels, that cannot be realized for a wide range of stimulus parameters in biological or technical systems. Srinivasan and Bernard ${ }^{90}$ proposed another cellular mechanism for a multiplication of neural signals that is related in some respects to the above-mentioned threshold model. A neuron is assumed to fire only in response to the coincident arrivals of spikes from two input neurons. Of course, such an element does not represent a multiplier with a secondorder nonlinearity. Instead, the second-order behavior will emerge only if either the time-averaged activity of a single 
element or, alternatively, the mean output of a large number of statistically independent elements is taken into account. ${ }^{90}$ Of course, at the present time we cannot exclude this possibility. With the slit paradigm in combination with pharmacological and electrophysiological techniques, it now seems to be possible to analyze the cellular implementation of the essential nonlinearity in the fly's motion-detection system.

\section{ACKNOWLEDGMENTS}

We thank K. G. Götz and J. Zanker for many useful comments on the manuscript. We also thank S. Marcinowski and F. Buchstäber for drawing the figures and I. Geiss for secretarial assistance.

\section{REFERENCES}

1. T. Poggio and W. Reichardt, "Considerations on models of movement detection," Kybernetik 13, 223-227 (1973).

2. E. Buchner, "Elementary movement detectors in an insect visual system," Biol. Cybern. 24, 85-101 (1976).

3. J. O. Limb, and J. A. Murphy, "Estimating the velocity of moving images in television signals," Comput. Graphics Image Process. 4, 311-327 (1975).

4. B. K. P. Horn and B. G. Schunck, "Determining optical flow," Artif. Intell. 17, 185-203 (1981).

5. D. Marr and S. Ullman, "Directional selectivity and its use in early visual processing," Proc. R. Soc. London Ser. B 223, 151180 (1981).

6. B. Moulden and H. Begg, "Some tests of the Marr-Ullman model of movement detection," Perception 15, 139-155 (1986).

7. E. C. Hildreth and C. Koch, "The analysis of visual motion: from computational theory to neuronal mechanisms," Annu. Rev. Neurosci. 10, 477-533 (1987).

8. W. Reichardt, "Autocorrelation, a principle for evaluation of sensory information by the central nervous system," in Principles of Sensory Communication, W. A. Rosenblith, ed. (Wiley, New York, 1961), pp. 303-317.

9. W. Reichardt, "Evaluation of optical motion information by movement detectors," J. Comp. Physiol. 161, 533-547 (1987).

10. J. Thorson, "Small-signal analysis of a visual reflex in the locust. I. Input parameters," Kybernetik 3, 4-52 (1966).

11. J. Thorson, "Small-signal analysis of a visual reflex in the locust. II. Frequency dependence," Kybernetik 3, 53-66 (1966).

12. K. Kirschfeld, "The visual system of Musca: studies on optics, structure and function," in Information Processing in the Visual Systems of Arthropods, R. Wehner, ed. (Springer-Verlag, Berlin, 1972), pp. 61-74.

13. D. H. Foster, "A model of the human visual system in its response to certain classes of moving stimuli," Kybernetik 8, 6984 (1971).

14. A. J. van Doorn and J. J. Koenderink, "Temporal properties of the visual detectability of moving spatial white noise," Exp. Brain Res. 45, 179-188 (1982).

15. A. J. van Doorn and J. J. Koenderink, "Spatial properties of the visual detectability of moving white noise," Exp. Brain Res. 45, 189-195 (1982).

16. J. P. H. van Santen and G. Sperling, "Temporal covariance model of human motion perception," J. Opt. Soc. Am. A 1, 451473 (1984).

17. J. P. H. van Santen and G. Sperling, "Elaborated Reichardt detectors," J. Opt. Am. A 2, 300-321 (1985).

18. H. R. Wilson, "A model for direction selectivity in threshold motion perception," Biol. Cybern. 51, 213-222 (1985).

19. E. H. Adelson and J. R. Bergen, "Spatiotemporal energy models for the perception of motion," J. Opt. Soc. Am. A 2, 284-299 (1985).

20. B. Hassenstein and W. Reichardt, "Systemtheoretische Analyse der Zeit-, Reihenfolgen- und Vorzeichenauswertung bei der Bewegungsperzeption des Rüsselkäfers Chlorophanus," Z. Naturforsch. B 11, 513-524 (1956).
21. W. Reichardt, "Autokorrelations-Auswertung als Funktionsprinzip des Zentralnervensystems (bei der optischen Wahrnehmung eines Insektes)," Z. Naturforsch. B 12, 448-457 (1957).

22. W. Reichardt and D. Varjú, "Übertragungseigenschaften im Auswertesystem für das Bewegungssehen (Folgerungen aus Experimenten an dem Rüsselkäfer Chlorophanus viridis)," $\mathrm{Z}$. Naturforsch. B 14, 674-689 (1959).

23. D. Varjú, "Optomotorische Reaktionen auf die Bewegung periodischer Helligkeitmuster (Anwendung der Systemtheorie auf Experimente am Rüsselkäfer Chlorophanus viridis)," Z. Naturforsch. B 14, 724-735 (1959).

24. K. G. Götz, "Optomotorische Untersuchung des visuellen Systems einiger Augenmutanten der Fruchtfliege Drosophila," Kybernetik 2, 77-92 (1964).

25. E. Buchner, "Behavioural analysis of spatial vision in insects," in Photoreception and Vision in Invertebrates, M. A. Ali, ed. (Plenum, New York, 1984), pp. 561-621.

26. N. M. Grzywacz and C. Koch, "Functional properties of models for direction selectivity in the retina," Synapse 1, 417-434 (1987).

27. B. Hassenstein, "Optokinetische Wirksamkeit bewegter periodischer Muster," Z. Naturforsch B 14, 659-674 (1959).

28. K. G. Götz, "Principles of optomotor reactions in insects," Bibl. Ophthalmol. 82, 251-259 (1972).

29. G. Zimmermann, "Der Einfluss stehender und bewegter Musteranteile auf die optomotorische Reaktion der Fliege Drosophila," doctoral dissertation (Eberhard-Karls-Universität Tübingen, Tübingen, Federal Republic of Germany, 1973).

30. T. Poggio and W. Reichardt, "Visual control of orientation behaviour in the fly. Part II. Towards the underlying neural interactions," Q. Rev. Biophys. 9, 377-438 (1976).

31. P. Kunze, "Untersuchung des Bewegungssehens fixiert fliegender Bienen," Z. Vergl. Physiol. 44, 656-684 (1961).

32. G. D. McCann and G. F. MacGinitie, "Optomotor response studies of insect vision," Proc. R. Soc. London Ser. B 163, 369 401 (1965).

33. H. Eckert, "Optomotorische Untersuchungen am visuellen System der Stubenfliege Musca domestica L," Kybernetik 14, 1-23 (1973).

34. W. Reichardt and A. Guo, "Elementary pattern discrimination (behavioural experiments with the fly Musca domestica),"Biol. Cybern. 53, 285-306 (1986).

35. D. J. Tolhurst, "Separate channels for the analysis of the shape and the movement of a moving visual stimulus," J. Physiol. 231, 385-402 (1973).

36. A. Pantle, "Motion aftereffect magnitude as a measure of the spatio-temporal response properties of direction-sensitive analyzer," Vision Res. 14, 1229-1236 (1974).

37. H. C. Diener, E. R. Wist, J. Dichgans, and T. Brandt, "The spatial frequency effect on perceived velocity," Vision Res. 16, 169-176 (1976).

38. D. H. Kelly, "Motion and vision. II. Stabilized spatio-temporal threshold surface," J. Opt. Soc. Am. 69, 1340-1349 (1979).

39. D. C. Burr and J. Ross, "Contrast sensitivity at high velocities," Vision Res. 22, 479-484 (1982).

40. S. J. Anderson and D. C. Burr, "Spatial and temporal selectivity of the human motion detection system," Vision Res. 8, 11471154 (1984).

41. W. J. Wright and A. Johnston, "Invariant tuning of motion aftereffect," Vision Res. 25, 1947-1955 (1985).

42. R. Hengstenberg, and K. G. Götz, "Der Einfluss des Schirmpigmentgehalts auf die Helligkeits- und Kontrastwahrnehmung bei Drosophila-Augenmutanten," Kybernetik 3, 276-285 (1967).

43. B. P. M. Lenting, H. A. K. Mastebroek, and W. H. Zaagman, "Saturation in a wide-field, directionally selective movement detection system in fly vision," Vision Res. 24, 1341-1347 (1984).

44. M. Egelhaaf and A. Borst, "Transient and steady-state response properties of movement detectors," J. Opt. Soc. Am. A 6, 116127 (1989).

45. M. Egelhaaf and W. Reichardt, "Dynamic response properties of movement detectors: theoretical analysis and electrophysiological investigation in the visual system of the fly," Biol. Cybern. 56, 69-87 (1987). 
46. W. Reichardt and M. Egelhaaf, "Properties of individual movement detectors as derived from behavioural experiments on the visual system of the fly," Biol. Cybern. 58, 287-294 (1988).

47. K. Hausen, "Motion sensitive interneurons in the optomotor system of the fly. I. The horizontal cells: structure and signals," Biol. Cybern. 45, 143-156 (1982).

48. W. H. Zaagman, H. A. K. Mastebroek, and J. W. Kuiper, "On the correlation model: performance of a movement detecting neural element in the fly visual system," Biol. Cybern. 31, 163168 (1978).

49. H. A. K. Mastebroek, W. H. Zaagman, and B. P. M. Lenting, "Movement detection: performance of a wide-field element in the visual system of the blowfly," Vision Res. 20, 467-474 (1980).

50. M. Egelhaaf, "On the neuronal basis of figure-ground discrimination by relative motion in the visual system of the fly. III. Possible input circuitries and behavioural significance of the FD-cells," Biol. Cybern. 52, 267-280 (1985).

51. R. R. de Ruyter van Steveninck, W. H. Zaagman, and H. A. K. Mastebroek, "Adaptation of transient responses of a movement-sensitive neuron in the visual system of the blowfly Calliphora erythrocephala," Biol. Cybern. 54, 223-236 (1986).

52. A. Borst and M. Egelhaaf, "Temporal modulation of luminance adapts time constant of fly movement detectors," Biol. Cybern. 56, 209-215 (1987).

53. H. Bülthoff and K. G. Götz, "Analogous motion illusion in man and fly," Nature (London) 278, 636-638 (1979).

54. R. D. DeVoe, "Movement sensitivities of cells in the fly's medulla," J. Comp. Physiol. 138, 93-119 (1980).

55. P. H. Marmarelis and G. D. McCann, "Development and application of white-noise modeling techniques for studies of insect visual nervous system," Kybernetik 12, 74-89 (1973).

56. G. D. McCann, "Nonlinear identification theory models for successive stages of visual nervous systems of flies," J. Neurophysiol. 37, 869-895 (1974).

57. H. B. Barlow and W. R. Levick, "The mechanism of directionally selective units in rabbit's retina," J. Physiol. 178, 477-504 (1965).

58. O.-J. Grüsser and U. Grüsser-Cornehls, "Neuronal mechanisms of visual movement perception and some psychophysical and behavioral correlations," in Handbook of Sensory Physiology, R. Jung, ed. (Springer-Verlag, Berlin, 1973), Vol. 7, Sec. 3A, pp. 333-429.

59. C. L. Baker and M. S. Cynader, "Spatial receptive-field properties of direction-selective neurons in cat striate cortex," J. Neurophysiol. 55, 1136-1152 (1986).

60. C. L. Baker and M. S. Cynader, "Space-time separability of direction selectivity in cat striate cortex neurons," Vision Res. 28, 239-246 (1988).

61. A. Mikami, W. R. Newsome, and R. H. Wurtz, "Motion selectivity in macaque visual cortex. I. Mechanisms of direction and speed selectivity in extrastriate area MT," J. Neurophysiol. 55, 1308-1327 (1986).

62. A. Mikami, W. T. Newsome, and R. H. Wurtz, "Motion selectivity in macaque visual cortex: II. Spatiotemporal range of directional interactions in MT and V1," J. Neurophysiol. 55, 1328-1339 (1986)

63. R. C. Emerson, M. C. Citron, W. J. Vaughn, and S. A. Klein, "Nonlinear directionally selective subunits in complex cells of cat striate cortex," J. Neurophysiol. 58, 33-65 (1987).

64. A. Riehle and N. Franceschini, "Motion detection in flies: parametric control over ON-OFF pathways," Exp. Brain Res. 54, 390-394 (1984).

65. N. Franceschini, "Early processing of colour and motion in a mosaic visual system," Neurosci. Res. Suppl. 2, 17-49 (1985).

66. F. H. Schuling "Processing of moving images in natural and artificial visual systems," doctoral dissertation (University of Groningen, Groningen, The Netherlands, 1988)

67. P. H. Schiller, B. L. Finlay, and S. F. Volman, "Quantitative studies of single-cell properties in monkey striate cortex. I. Spatiotemporal organization of receptive fields," J. Neurophysiol. 39, 1288-1319 (1976).
68. L. Ganz and R. Felder, "Mechanism of directional selectivity in simple neurons of the cat's visual cortex analyzed with stationary flash sequences," J. Neurophysiol. 51, 294-324 (1984).

69. J. D. Victor and R. M. Shapley, "Receptive field mechanisms of cat X and Y retinal ganglion cells," J. Gen. Physiol. 74, 275-298 (1979).

70. C. Enroth-Cugell, J. G. Robson, D. E. Schweitzer-Tong, and A. B. Watson, "Spatiotemporal interactions in cat retinal ganglion cells showing linear spatial summation," J. Physiol. 341, 279307 (1983).

71. M. V.Srinivasan and D. R. Dvorak, "Spatial processing of visual information in the movement-detecting pathway of the fly. Characteristics and functional significance," J. Comp. Physiol. 140, 1-23, (1980).

72. B. Pick, "Visual flicker induces orientation behaviour in the fly Musca," Z. Naturforsch. C 29, 310-312 (1974).

73. B. Pick, "Visual pattern discrimination as an element of the fly's orientation behaviour," Biol. Cybern. 23, 171-180 (1976).

74. G. Geiger and T. Poggio, "The orientation of flies towards visual patterns: on the search for the underlying functional interactions," Biol. Cybern. 19, 39-54 (1975).

75. W. Reichardt, "Functional characterization of neural interactions through an analysis of behavior," in The Neurosciences, Fourth Study Program, F. R. Schmitt and F. G. Worden, eds. (MIT Press, Cambridge, Mass., 1979), pp. 81-103.

76. H. Bülthoff and C. Wehrhahn, "Computation of motion and position in the visual system of the fly (Musca). Experiments with uniform stimulation," in Localization and Orientation in Biology and Engineering, D. Varjú and H. Schnitzler, eds. (Springer-Verlag, Berlin, 1984), pp. 149-152.

77. W. Reichardt and T. Poggio, "Visual control of orientation behaviour in the fly. Part I. A quantitative analysis," Q. Rev. Biophys. 9, 311-375 (1976).

78. L. B. Baker and O. J. Braddick, "Temporal properties of the short-range process in apparent motion," Perception 14, 181192 (1985).

79. P. H. Schiller, B. L. Finlay, and S. F. Volman, "Quantitative studies of single-cell properties on monkey striate cortex. III. Spatial frequency," J. Neurophysiol. 39, 1334-1351 (1976).

80. J. A. Movshon, I. D. Thompson, and D. J. Tolhurst, "Spatial summation in the receptive fields of simple cells in the cat's striate cortex;" J. Physiol. 283, 53-77 (1978).

81. J. A. Movshon, I. D. Thompson, and D. J. Tolhurst, "Receptive field organization of complex cells in the cat's striate cortex," J. Physiol. 283, 79-99 (1978).

82. L. Maffei, C. Morrone, M. Pirchio, and G. Sandini, "Responses of visual cortical cells to periodic and non-periodic stimuli," J. Physiol. 296, 27-47 (1979).

83. R. L. De Valois, D. G. Albrecht, and L. G. Thorell, "Spatial frequency selectivity of cells in macaque visual cortex," Vision Res. 22, 545-559 (1982).

84. K. G. - Götz, "Die optischen Übertragungseigenschaften der Komplexaugen von Drosophila," Kybernbetik 2, 215-221 (1965).

85. R. A. Holub and M. Morton-Gibson, "Response of visual cortical neurons of the cat to moving sinusoidal gratings: responsecontrast functions and spatiotemporal interactions," J. Neurophys. 46, 1244-1259 (1981).

86. M. C. Morrone, M. di Stefano, and D. C. Burr, "Spatial and temporal properties of neurons of the lateral suprasylvian cortex of the cat," J. Neurophysiol. 56, 969-986 (1986).

87. D. H. Hubel and T. N. Wiesel, "Receptive fields, binocular interaction, and functional architecture in the cat's visual cortex," J. Physiol. 160, 106-154 (1962)

88. H. Spitzer and S. Hochstein, "A complex-cell receptive-field model," J. Neurophysiol. 53, 1266-1286 (1985).

89. V. Torre and T. Poggio, "A synaptic mechanism possibly underlying directional selectivity to motion," Proc. R. Soc. London Ser. B 202, 409-416 (1978).

90. M. V. Srinivasan and G. D. Bernard, "A proposed mechanism for multiplication of neural signals," Biol. Cybern. 21, 227-236 (1976). 